\title{
Internal Tide and Nonlinear Internal Wave Behavior at the Continental Slope in the Northern South China Sea
}

\author{
Timothy F. Duda, James F. Lynch, Senior Member, IEEE, James D. Irish, Robert C. Beardsley, Steven R. Ramp, \\ Ching-Sang Chiu, Tswen Yung Tang, and Ying-Jang Yang
}

\begin{abstract}
A field program to measure acoustic propagation characteristics and physical oceanography was undertaken in April and May 2001 in the northern South China Sea. Fluctuating ocean properties were measured with 21 moorings in water of 350to 71-m depth near the continental slope. The sea floor at the site is gradually sloped at depths less than $90 \mathrm{~m}$, but the deeper area is steppy, having gradual slopes over large areas that are near critical for diurnal internal waves and steep steps between those areas that account for much of the depth change. Large-amplitude nonlinear internal gravity waves incident on the site from the east were observed to change amplitude, horizontal length scale, and energy when shoaling. Beginning as relatively narrow solitary waves of depression, these waves continued onto the shelf much broadened in horizontal scale, where they were trailed by numerous waves of elevation (alternatively described as oscillations) that first appeared in the continental slope region. Internal gravity waves of both diurnal and semidiurnal tidal frequencies (internal tides) were also observed to propagate into shallow water from deeper water, with the diurnal waves dominating. The internal tides were at times sufficiently nonlinear to break down into bores and groups of high-frequency nonlinear internal waves.
\end{abstract}

Index Terms-Baroclinic tides, internal waves, nonlinear waves.

\section{INTRODUCTION}

I N APRIL and May 2001, a suite of physical oceanographic moorings was deployed in the northern portion of the South China Sea (SCS) as part of the Asian Seas International Acoustics Experiment (ASIAEX) South China Sea 2001 acoustic volume interaction study. This was a joint physical oceanographic/ocean acoustics program. The moorings measured time series of temperature, conductivity, and current velocity throughout the water column for the dual purposes of studying the local ocean physics and directly comparing temporal and spatial variability of acoustical and environmental signals. The direct comparisons can be found in two companion papers [1], [2].

Two categories of internal gravity waves dominate the data from the moorings. The first category consists of large-ampli-

Manuscript received June 6, 2003; revised August 11, 2003. This work was supported in part by grants from the U.S. Office of Naval Research, Physical Oceanography and Ocean Acoustics Programs, and by the National Science Council of Taiwan.

T. F. Duda, J. F. Lynch, J. D. Irish, and R. C. Beardsley are with the Woods Hole Oceanographic Institution, Woods Hole, MA 02543 USA (e-mail: tduda@whoi.edu; jlynch@whoi.edu).

S. R. Ramp and C.-S. Chiu are with the Naval Postgraduate School, Monterey, CA 93943 USA.

T. Y. Tang is with the National Taiwan University, Taipei, Taiwan.

Y.-J. Yang is with the Chinese Naval Academy, Kaohsiung, Taiwan.

Digital Object Identifier 10.1109/JOE.2004.836998 tude solitary-type waves of depression coming from the east that appear to emanate from the vicinity of the Luzon Strait [3]. These are called transbasin waves because they cross the northern part of the SCS. The second category consists of waves at or near tidal frequencies, which we call the internal tide. In this paper we will show the basic properties at this site of each of these two types of waves.

The transbasin waves may be the largest (highest amplitude) internal gravity waves of quasi-permanent solitary form ever measured. Wave amplitudes of up to $90 \mathrm{~m}$ in the Sulu Sea have been reported [4], whereas we observe waves of 160-m amplitude or greater in the SCS. Many properties of the transbasin waves derived from these mooring observations are covered in a companion paper by Ramp et al. [5]. These waves were also studied by Orr and Mignerey from a ship during ASIAEX using high-frequency acoustic imaging, Doppler current profiling, and towed CTD [6]. A fourth paper by Liu et al. compares the observed waves with theory [7].

The transbasin waves appeared regularly during the ASIAEX SCS 2001 field program, with an approximate fortnightly cycle, arriving in two 9-d-long groups of wave packets separated by a 5-d-long period without waves. Ramp et al. explain the observed wave packets and groups in detail, and some of their results are briefly summarized here. (They refer to these groups as clusters.) The first wave group was in the period April 22 to May 1. The second group was in the period May 6-14. In each group, wave amplitudes build for a few days, reach a peak, and then decline over the last few days. The between-packet interval within the groups is about $12 \mathrm{~h}$. Once each day, at roughly the same time in the morning (UTC), a type- $a$ packet having one initial large wave arrives. Between the type-a packets come type- $b$ packets, which have less-regular wave amplitudes and wave timing structure than type a. Type-a and type-b packets are therefore interleaved. Both packet types contain nonlinear internal waves arriving from the east; they differ only in detail: the type-a packets arrive at 24-hr interval and have an initial wave larger than those following; the type-b packets arrive at 25- to 26-hr interval and are not led by the largest wave.

The transbasin waves propagate from deep to shallow water through the study area. We will show how they respond to the shoaling of the sea floor, which would force wave energy density to increase in the absence of dissipation. There are already many published results concerning nonlinear wave evolution over a shoaling bottom [8]-[11]. In particular, building on the observations of Orr and Mignerey collected between the 110- and 260-m 


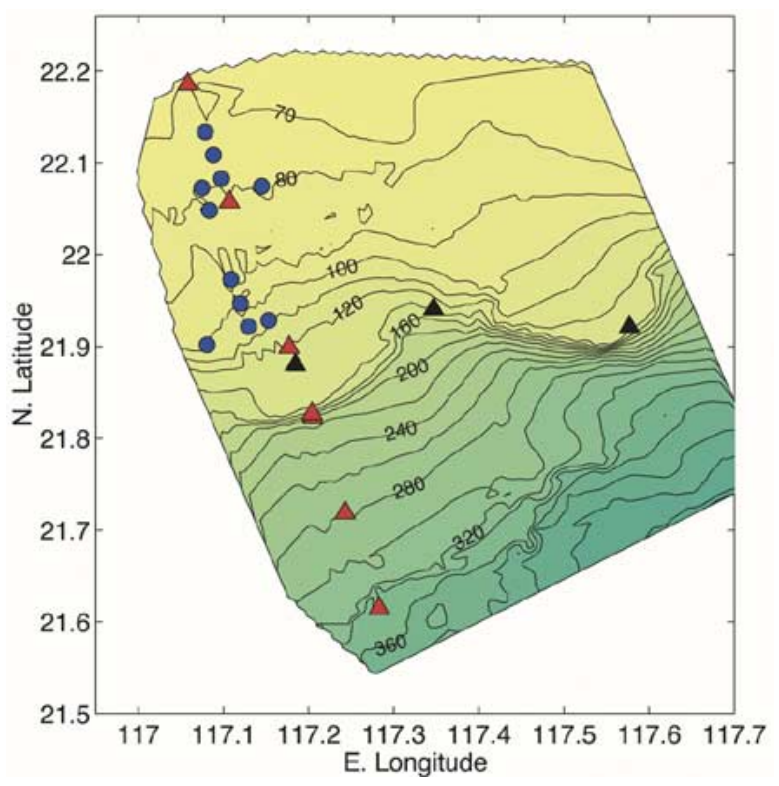

Fig. 1. Contoured bathymetry and ASIAEX mooring locations are shown. Depth is in meters. Environmental moorings with ADCPs are shown in red, designated S2-S7 from the top (quantity 6). Vertical strings of thermometers are shown in black (3). Loco moorings with three thermometers each are shown in blue (11).

isobaths, we show here that the transbasin solitons widen, slow down, and lose energy, but do not appreciably refract (within our ability to measure wave direction) as they move from 350- to 80-m water depth. Orr and Mignerey found one wave arriving in the morning (UTC) of May 8 (also reported on here) to contain $57 \mathrm{MJ} / \mathrm{m}$ kinetic energy in water of 260-m depth, diminishing after $16 \mathrm{~km}$ of propagation to $16 \mathrm{MJ} / \mathrm{m}$ in water of $110-\mathrm{m}$ depth. They also estimated the average dissipation rate at these water depths for the entire packet of one-half dozen or so waves to be $0.17 \mathrm{~W} / \mathrm{m}^{2}$. We do not compare the wave evolution with nonlinear wave evolution theories in this paper, in part because the dissipation is not handled well by the theories.

The internal tide is also very pronounced at this site, and we will examine its relation to barotropic tidal currents measured at the same time [12]. The barotropic tides in the area have significant energy in four constituents: Principal lunar (O1) and lunisolar (K1), both diurnal, plus principal lunar (M2) and principal solar $(\mathrm{S} 2)$, both semidiurnal. The periods are 25.82, $23.94,12.42$, and $12.00 \mathrm{~h}$. Waves of these periods enter the SCS through the Luzon Strait and propagate in a southwesterly direction, some resonating in the southern SCS, and the M2 resonating north of our site in the Taiwan Strait [13]. We show that diurnal, and to a lesser extent semidiurnal, internal tides both exist, and that the diurnal waves dominate the isotherm displacement field during the 5-d gaps between the transbasin wave groups, particularly in water depths less than $200 \mathrm{~m}$. There are sufficient data to compute diurnal internal tidal horizontal wavelengths, vertical wavelengths, horizontal phase velocities, and energy fluxes. Evidence is presented that bottom slope and tidal constituent interference combine to favor diurnal over semidiurnal internal tidal wave generation at the ASIAEX site.

The paper is organized as follows. Section II describes the experimental area, the moored instrumentation, and data processing methods. Data illustrating the internal wave conditions

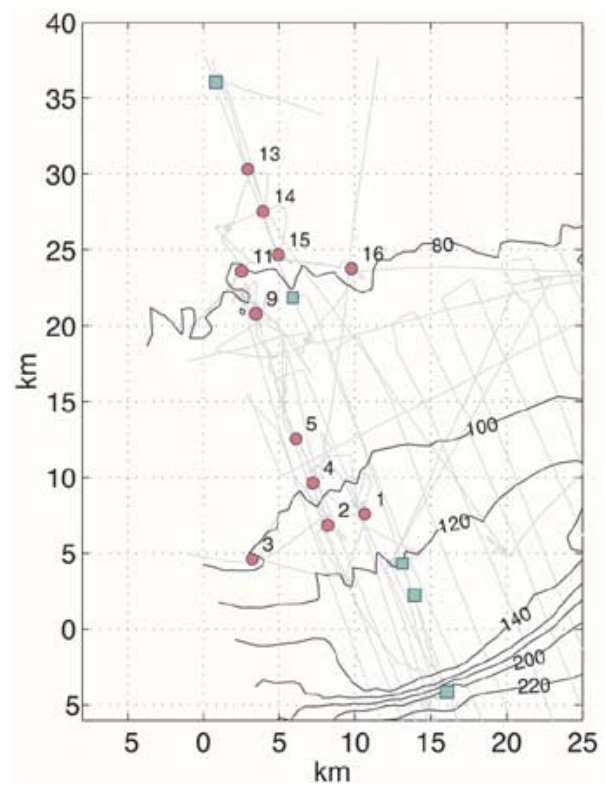

Fig. 2. Shallow-water moorings and bathymetry are shown using a kilometer scale. Depths are in meters. The Loco moorings are shown with red circles and the Loco mooring designation numbers are indicated. The moorings S2-S5a with ADCPs are shown with the squares, with an additional square at 124-m depth indicating the position of the acoustic receiver array that was equipped with a vertical line of thermometers.

TABLE I

Distances and Headings Between Selected MOORING PAIRS (KM/DEGREES)

\begin{tabular}{lccccc}
\hline- & S5 & S5a & S4 & S3 & S2 \\
\hline S7 & $24.38 / 340.8$ & $24.90 / 340.9$ & $33.36 / 340.8$ & $52.31 / 339.7$ & $67.37 / 339.9$ \\
S5 & - & $0.52 / 343.0$ & $8.98 / 340.9$ & $27.93 / 338.7$ & $43.00 / 339.3$ \\
S5a & - & - & $8.46 / 340.7$ & $27.42 / 338.6$ & $42.48 / 339.3$ \\
S4 & - & - & - & $18.97 / 337.6$ & $34.02 / 338.9$ \\
S3 & - & - & - & - & $15.07 / 340.5$ \\
\hline
\end{tabular}

are presented in Section III. Section IV examines the shoaling behavior of a few selected nonlinear transbasin waves. Section V contains a discussion of the wave physics. Section VI examines the behavior of internal (baroclinic) tides fitted to current and displacement time series. Section VII discusses the diurnal waves that dominate the internal tides in the region. Section VIII is a summary of the results.

\section{EXPERIMENTAL REGION AND METHODS}

An array of 32 moorings carrying physical oceanographic sensors was deployed around April 21, 2001. Twenty-one of them at twenty sites were successfully recovered (Fig. 1), all around May 21, except for one mooring at 85-m depth that was damaged on May 5 and recovered in two pieces. Only the recovered moorings are shown in the figure. Eleven were low-cost (Loco) moorings carrying three temperature sensors and one pressure sensor each. The Locos were spaced 2-3 km from one another and formed two groups, southern and northern, each in the vicinity of an ADCP. Seven Loco moorings, mostly deployed in the zone between the two groups, were not recovered and may have been lost to fishing activity. Fig. 2 provides 


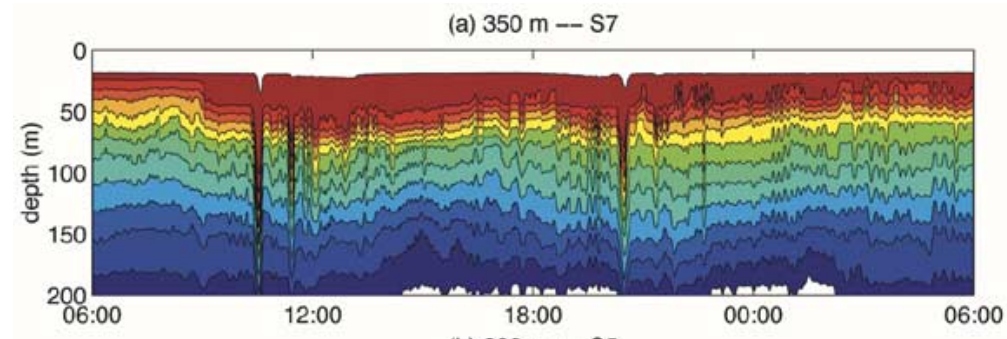

(b) $200 \mathrm{~m}-\mathrm{S} 5$

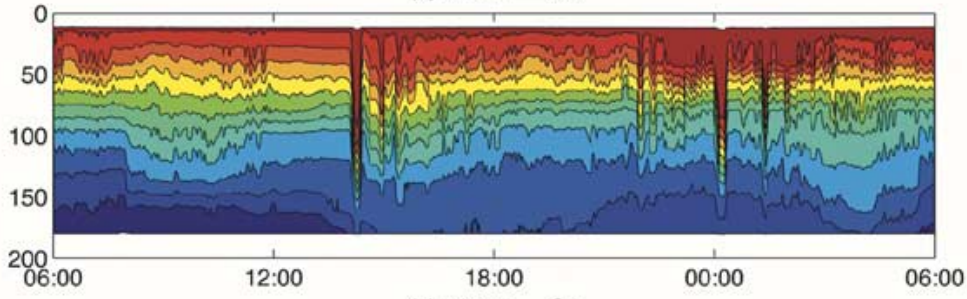

(c) $120 \mathrm{~m}-\mathrm{S} 4$

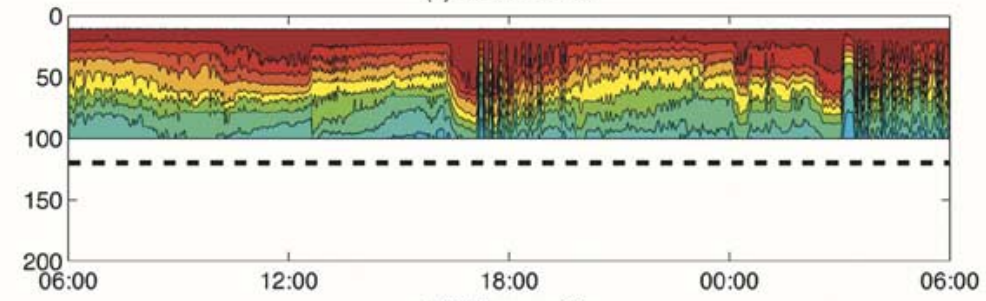

(d) $85 \mathrm{~m}-\mathrm{S} 3$

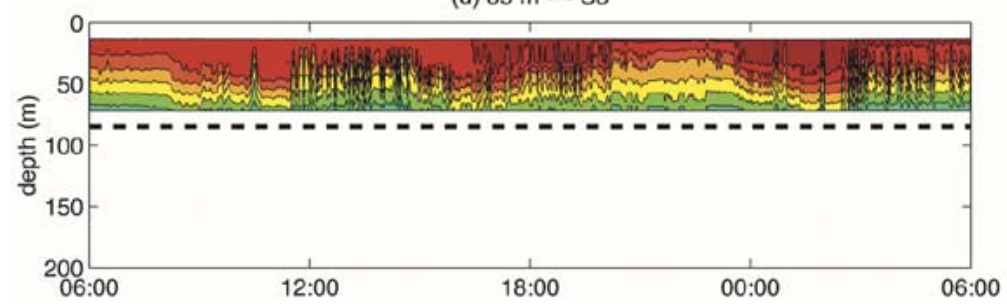

Fig. 3. Contours of 24-h time series of $T(z, t)$ from four sites beginning at 0600 UTC April 23 . The contour interval is $1^{\circ}$. The deepest blue color is the $15-16^{\circ}$ range. (a) Data from the upper $200 \mathrm{~m}$ at site S7 are shown. (b) Data from site S5 are shown. (c) Data from S4 are shown, with the bottom depth indicated with the dashed line. (d) Data from S3 are shown, with the bottom depth again indicated with a dashed line.

the Loco mooring numbers and shows depth contours in the shallow area. Ship tracks where depth data were collected are also shown. Fig. 1 shows the terraced nature of the bottom. Gradual slopes of approximately $0.3^{\circ}$ dominate the area, but there are isolated steep areas with dropoffs of 60-100 m occurring over short distances of hundreds of meters or less.

More heavily instrumented moorings occupied six sites along a line of heading $340^{\circ}$. These were designated S2-S7 going from shallow to deep water. Their depths were $71,85,120$, 200, 275, and $350 \mathrm{~m}$, respectively. Fig. 1 shows these in red. These had the same sensors as the Locos, although many more of them, plus current meters and conductivity sensors. Two sites (S7 and S3) also had precision pressure gauges near the bottom for sensing waves and tides. S3 is the mooring that was damaged on May 5. The instrument depths in the water column can be found in Ramp et al. [5]. S5 had its current meter on a separate nearby mooring called S5a. Table I gives exact distances and headings between these moorings. Vertical thermometer arrays were also deployed at three other positions. One additional large mooring to the east of the line was lost (S1).

All current meters were acoustic Doppler current profilers (ADCPs) except for three rotor-type instruments placed deep at the 350-m site. The ADCPs pinged at a 1-s rate and recorded averages every $2 \mathrm{~min}$. The depth-bin spacing was $8 \mathrm{~m}$ for the S5a instrument and $4 \mathrm{~m}$ for the others. The other current meters recorded vector averages, also at 2-min interval. The temperature, conductivity, and water column pressure gauges sampled at $30-, 60-$, or $120-$ s intervals. The precision near-bottom pressure gauges sampled once per hour with additional high-rate bursts for wave sensing.

Internal wave parameters are computed from two types of data: isotherm displacement time series $\eta_{T}(t)$, where subscript $(T=18,20$, etc.) indicates isotherm temperature, and current time series $V(z, t)=u+i v$, with $u$ eastward and $v$ northward. Displacements are calculated by linear interpolation vertically between temperature time series $T(t, z)$ recorded at the individual sensors. The time-dependent vertical positions of the sensors were estimated from the pressure records collected along the mooring cables. The sensor depths fluctuate tens of meters, but are known to within 1 or $2 \mathrm{~m}$. The current time series are treated as though the instrument depths are stable and, thus, measure shear better than they do velocity time series at specific depths. There are also appreciable mooring tilts caused by strong currents, possibly up to $30^{\circ}$, which create errors in ADCP current estimates. ADCP error also increases during passage of the shortest nonlinear waves, which produce different velocities 

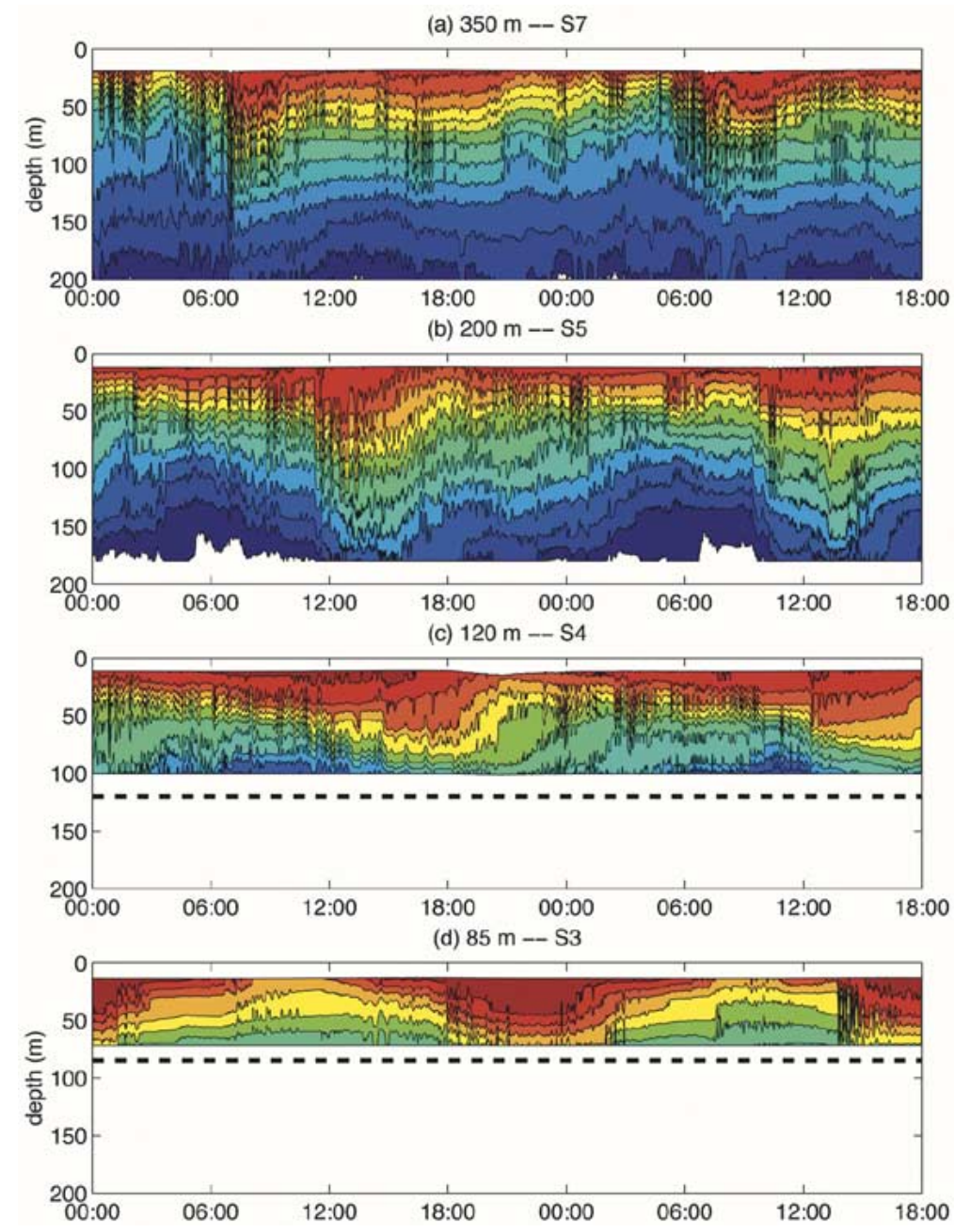

Fig. 4. Contours of $42 \mathrm{~h}$ of $T(z, t)$ data from May 2 and 3 at four sites. The contour interval is $1^{\circ}$. (a) Data from the upper $200 \mathrm{~m}$ at site S7. (b) Data from S5. (c) Data from S4, with the bottom depth indicated with a dashed line. (d) Data from S3, with the bottom depth indicated with a dashed line.

in each of the four diverging beams, a violation of assumptions that are made when computing velocity vectors from the slant velocities.

Harmonic tidal analysis was performed with the T_Tide program [14] for the purpose of extracting internal tides from the data. T_Tide is a code based on earlier published algorithms but with updated error analysis. The regular arrival of transbasin wave packets at roughly the semidiurnal tidal period complicates the tidal analysis and must be dealt with. Periodic packets do not have the same dynamics as the internal waves of tidal frequency that we wish to isolate, but they do influence the tidal least-squares fitting procedure for isotherm height. Fitting to the complex velocity field $V$ is less sensitive to this contamination because the high-frequency transbasin wave velocities are rectilinear and the internal tidal currents are orbital. Transbasin wave velocities and displacements are eliminated from the analysis as follows: hourly mean currents and isotherm heights are computed and hourly bins with variance exceeding an empirically determined threshold are then eliminated from consideration, creating gappy time series that are then analyzed. Typically, 5\% percent of data from the near-bottom depths are eliminated, $15 \%$ at middle depths, and up to $35 \%$ at the shallowest depths.

\section{INTERNAL WAVE "WEATHER"}

Figs. 3 and 4 show some features of the internal gravity wave "weather." We call this wave weather because we have only 1 mo of observations, so we do not know the complete wave climate. Figures in the Ramp et al. paper [5] show the 1-mo records of wave activity, encompassing the two 9-d long periods of transbasin wave activity. Fig. 3, showing data of April 23, shows the passage of one large transbasin wave from a type-a packet into shallow water. The wave passes the 350-m depth mooring (S7) at 1030 UTC, is again evident at the 200-m depth mooring (S5) about 4-5 h later near 1400 UTC and is still evident at the $120-\mathrm{m}$ depth (S4) site near 1700 UTC. The wave takes a much longer time to pass by the 120-m site than the other two. We will show that the transbasin waves slow appreciably in this depth range and also broaden, with passage time lengthening due to both effects. There are also many more following waves at the $120-\mathrm{m}$ site than the deeper sites. At the 85-m site (S3), the transbasin wave expands to a very large width and bears little resemblance to its original form. A large wave in a type-b packet that passes S7 at 2100 UTC behaves similarly. The step-like features that develop at depths of $120 \mathrm{~m}$ and less are similar to those observed on the Australian North West Shelf at 80-m depth and modeled with some success using the extended Korteweg-de Vries 
(a) 8 May
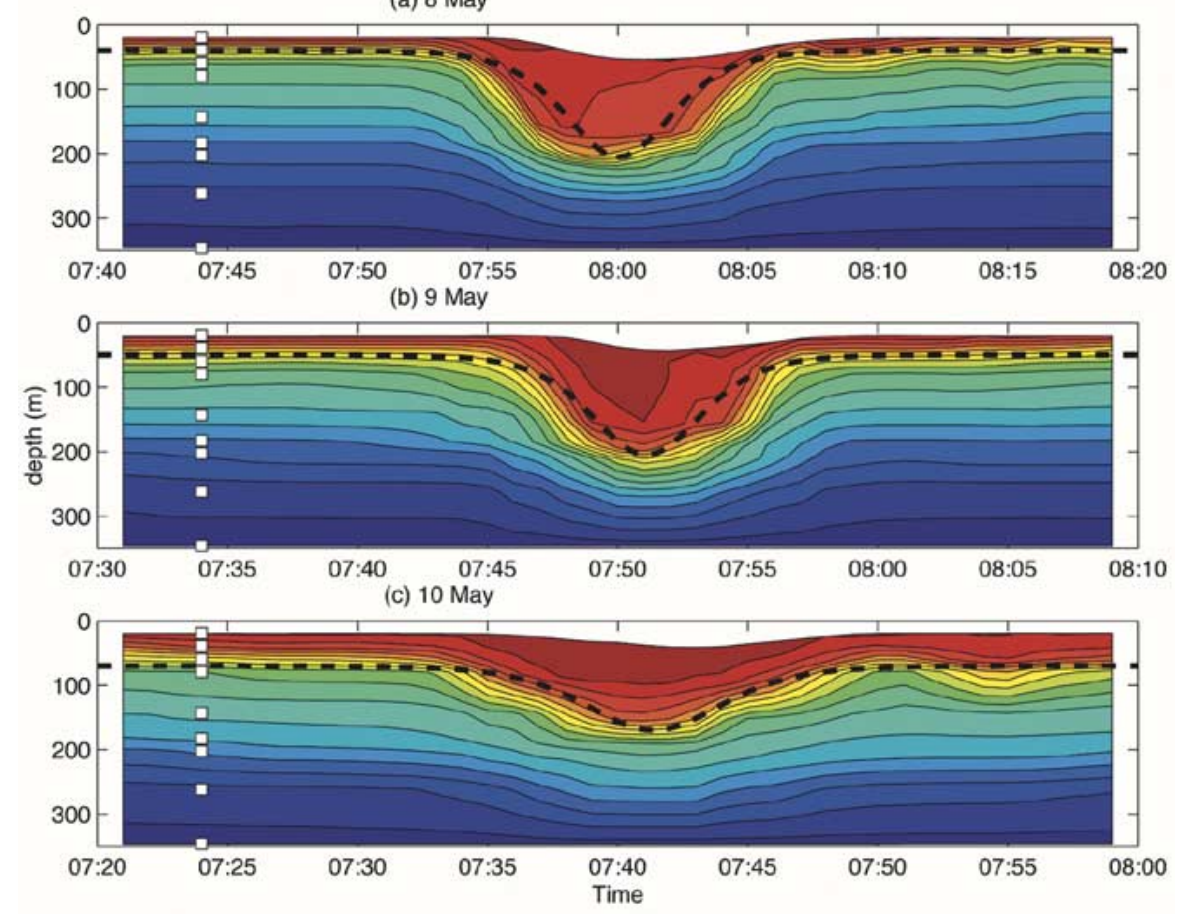

Fig. 5. Isotherms contoured for three consecutive large waves from type-a packets are shown. Shown are the waves from (a) May 8, (b) May 9, and (c) May 10. Sampling is once per minute. The black dashed lines show sech ${ }^{2}$ permanent form wave solutions of the KdV equation placed at an arbitrary depth. The contour intervals are $1^{\circ} \mathrm{C}$, with the darkest blue indicating temperatures in the $11-12^{\circ}$ range and the deep red indicating temperatures above $28^{\circ}$. The white squares show the mean depths of the thermometers that were used. The white patches at the center tops show the positions of the topmost sensor during the passage of the waves as the mooring was bent over by drag forces.

$(\mathrm{eKdV})$ equation [15]. Another interesting feature in Fig. 3 is the mode-two internal wave passing S5 just before 0800 UTC, which steepens to a mode-two bore passing S4 at about 1230 UTC. The numerous high-frequency internal waves passing S3 after 1600 UTC appear to be linked to the mode-two wave.

Fig. 4 shows $42 \mathrm{~h}$ of data from May 2-3 at the same four sites as shown in Fig. 3. This is a time period between the two groups of large transbasin waves, but there are still many short-wavelength nonlinear internal waves present. Note that the diurnal thermocline displacement exceeds $50 \mathrm{~m}$ at S5. The diurnal wave evolves into a bore at the 120-m depth site (S4), with solitary waves following, and continues on to S3 in this form. This is best seen at 1200 UTC of the second day at S4 and 1400 UTC at S3. Overall, the distinctive large transbasin waves occur in fortnightly groups, whereas other smaller short-scale internal waves and the long-scale internal tides are ubiquitous.

\section{ShOAling Nonlinear TRANSBASIN WAVE OBSERVATIONS}

The large transbasin waves were seen to undergo rapid changes as they encountered the slope. All evidence indicates that before they shoaled many of the waves had achieved (or nearly achieved) a permanent form, with nonlinear steepening balancing wave dispersion, thus allowing the waveform to remain stable (cf., [16]). An often-cited permanent-form example is the well-known hyperbolic secant squared solution of the KdV equation [16], [17]. Orr and Mignerey document the transition of single waves into groups of waves at depths between 260-110 $\mathrm{m}$ and the data shown herein agree with their observations: The quasi-permanent form of the waves is lost as they encounter the slope. In this section, we will examine waves from a 3-d period and describe in detail how the wave parameters that are reported were extracted from the data.

It is not surprising that the transbasin waves are dramatically altered at the slope, since they have amplitudes of $160 \mathrm{~m}$ or more at the 350-m depth site, yet still manage to continue onward into water of 80-m depth. Like large waves observed at other sites [18]-[20], these waves spawn additional waves (or oscillations) as they encounter changing bathymetry, changing stratification, and changing background vertical or horizontal shear, agreeing qualitatively with solutions of equations similar to the $\mathrm{KdV}$ equation [17]. The changing conditions, plus dissipation, prevent them from propagating with permanent form.

Fig. 5 shows isotherms for the three large initial type-a waves of May 8-10, measured at the 350-m deep S7 site. Orr and Mignerey sampled the May 8 wave and a similar wave on May 7.These initial waves of type-a groups appear in relative isolation from other large waves and may have permanent form, or at least propagate with only slight ongoing adjustments in order to equilibrate with changing conditions. The figure also shows $\mathrm{KdV}$ equation permanent form solutions $\eta(x, t)=a \operatorname{sech}^{2}[(x-c t) / L]$, with $a$ of $-165 ;-155$ and -100 $\mathrm{m} ; L$ of 221,228 , and $283 \mathrm{~m}$; and $c=1.27 \mathrm{~m} / \mathrm{s}$. The widths and amplitudes of $\mathrm{KdV} \operatorname{sech}^{2}$ solutions are related by $L^{2}=12 \gamma / \alpha a$, where $\gamma=7030$ and $\alpha=-0.0106$ are the dispersion and nonlinear coefficients of the $\mathrm{KdV}$ equation, each computed using an S7 density profile and the resulting vertical mode function [21].

The shapes of the May 9 and 10 waves are quite close to a $\operatorname{sech}^{2}$ form but are wider than is consistent with $\mathrm{KdV}$, indicating that these wave are not permanent form solutions of the weakly nonlinear KdV equation. The May 8 wave has a flatter peak 

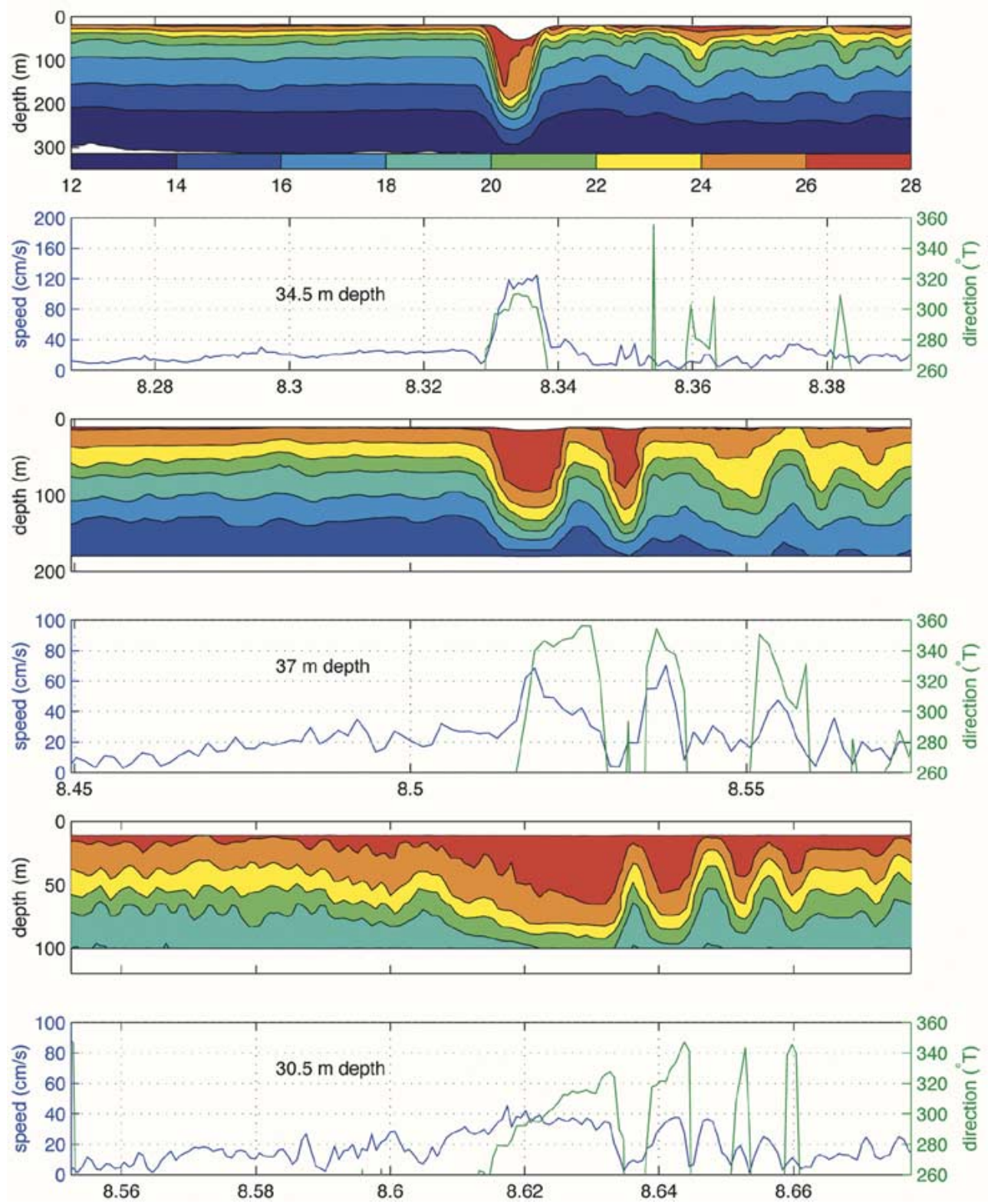

Fig. 6. The large initial type-a wave of May 8 is shown. The two top panels show data from S7, the middle two S5, and the lower two S4. Each panel pair shows $T(z, t)$ time series above and current speed and direction for an upper-ocean depth bin below. The time scale for each pair of panels in terms of date in May is given in the lower panel of each pair. The color scale for temperature contours is shown at the lower edge in the top panel. The upper-layer currents that are shown have been detided and then high-pass filtered. Time is in days, with $3 \mathrm{~h}$ shown in each panel.

than $\operatorname{sech}^{2}$. The departures from KdV wave shapes suggest that the waves may be better described by higher order equations (see [7], [20], and [22] and papers cited therein) and that watercolumn dissipation may play a role [23]. However, the large waves fit the KdV solutions better then may be expected given that the amplitudes are from 1.0 to 1.5 times the upper layer thickness, approximated by the peak of the first vertical mode function.

\section{A. May 8 Wave}

Orr and Mignerey have documented the decay of the May 8 wave shown in Figs. 5 and 6, plus one arriving $24 \mathrm{~h}$ earlier [6]. They followed the waves from 260- to 110-m depth. Here, we study properties of the May 8 wave at deeper and shallower areas, plus we study a May 10 wave for comparison.
These waves were chosen because detailed acoustic images of shear instability within the May 8 wave are presented by Orr and Mignerey and because they quantified kinetic energy loss over time in the wave. However, the May 8 wave exhibited anomalously slow phase velocity compared to the majority of waves, so we also study the May 10 wave that exhibited more typical behavior. Phase velocities of each wave at a few sites are derived here in detail.

Orr and Mignerey were able to follow waves in the direction of propagation (i.e., the direction normal to the wave crests) and, thus, were able to directly measure wave transformation. Such a measurement is not possible with our array of moorings, which was not aligned with the direction of propagation. It is evident from inconsistencies in the mooring data and from some of the available satellite SAR images that the waves do not always have 

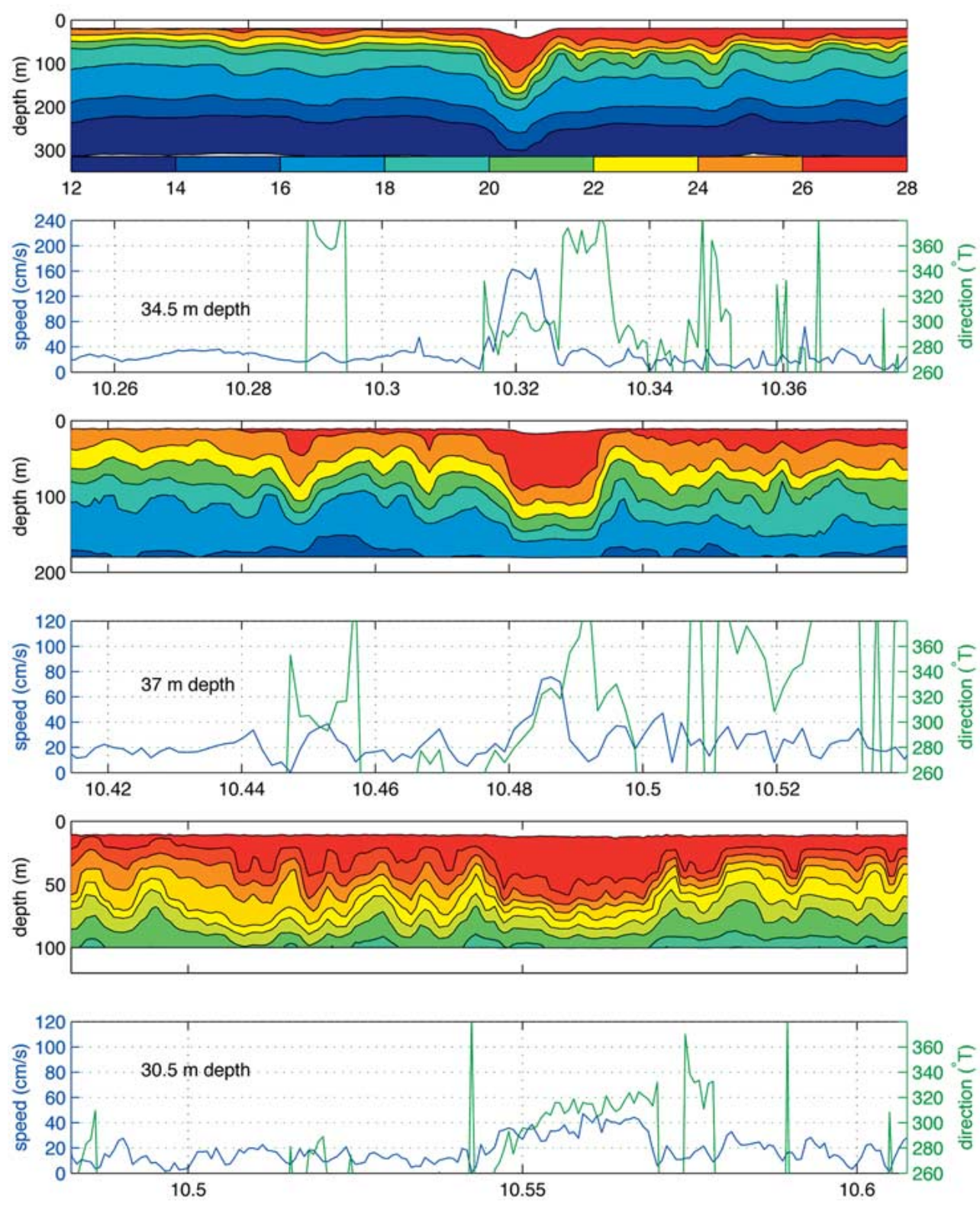

Fig. 7. Large initial May 10 type-a wave is shown in the same format as the previous figure. S7 data are shown in the top two panels, S5 and S5a data in the next two, and $\mathrm{S} 4$ data in the bottom two. $360^{\circ}$ has been added to direction values slightly to the east of north so that they appear on the figure.

linear crests (i.e., are not analogous geometrically to sinusoidal plane waves) and, therefore, are not amenable to simple fitting procedures. Wave speeds tabulated by Ramp et al. from mooring data have a wide range of values and some of the spread may be due to errors arising from wave crest curvature.

Fig. 6 shows data from the May 8 wave taken at S7, S5, and S4. Ramp et al. also show velocity time series from this wave at $\mathrm{S} 7$ for a few depths. This is the initial and largest wave of the morning (UTC) type-a packet of waves for this day. The top two panels show S7 data, the middle two show S5 and S5a data, and the lower two show S4 data. All panels show $3 \mathrm{~h}$ of data, with the initial downward pulse of the wave centered in the panel. The temperature time series at S7 (top panel) shows the solitary (and presumably quasi-permanent) nature of the wave at that site. Currents from an upper layer are shown in the second panel of each pair. A reliably estimated barotropic tidal current was removed [12] and the resultant series was high-pass filtered with an 8-h cutoff period to isolate wave velocities. The fitted barotropic tidal currents explain approximately $90 \%$ of the tidal-band variances of the depth-averaged velocities at each ADCP site. The barotropic tidal advection speeds are typically of order $10 \mathrm{~cm} / \mathrm{s}$. The $\mathrm{S} 7$ peak current speed of $120 \mathrm{~cm} / \mathrm{s}$ is directed at $300^{\circ}$ (true), indicating the current direction after removal of tidal advection, which is presumably normal to the crest. Skipping the high-pass filter step, which allows internal tidal signal to pass through the analysis, changes the direction to $290^{\circ}$.

At S5 to the north, there are two waves present. We assume the first and larger wave to be the one observed at S7. Both waves are directed toward $340^{\circ}$, a northward shift of $40^{\circ}$. Based on a travel direction of $300^{\circ}$ at $\mathrm{S} 7$ and the mooring line heading of $340^{\circ}$ (i.e., the wave is not propagating along the line connecting the moorings), the portion of the first wave passing S5 is likely to have passed $10 \mathrm{~km}$ to the north of S7; thus, the creation of the second wave in the 4.5-h interval between S5 and S7 wave pas- 


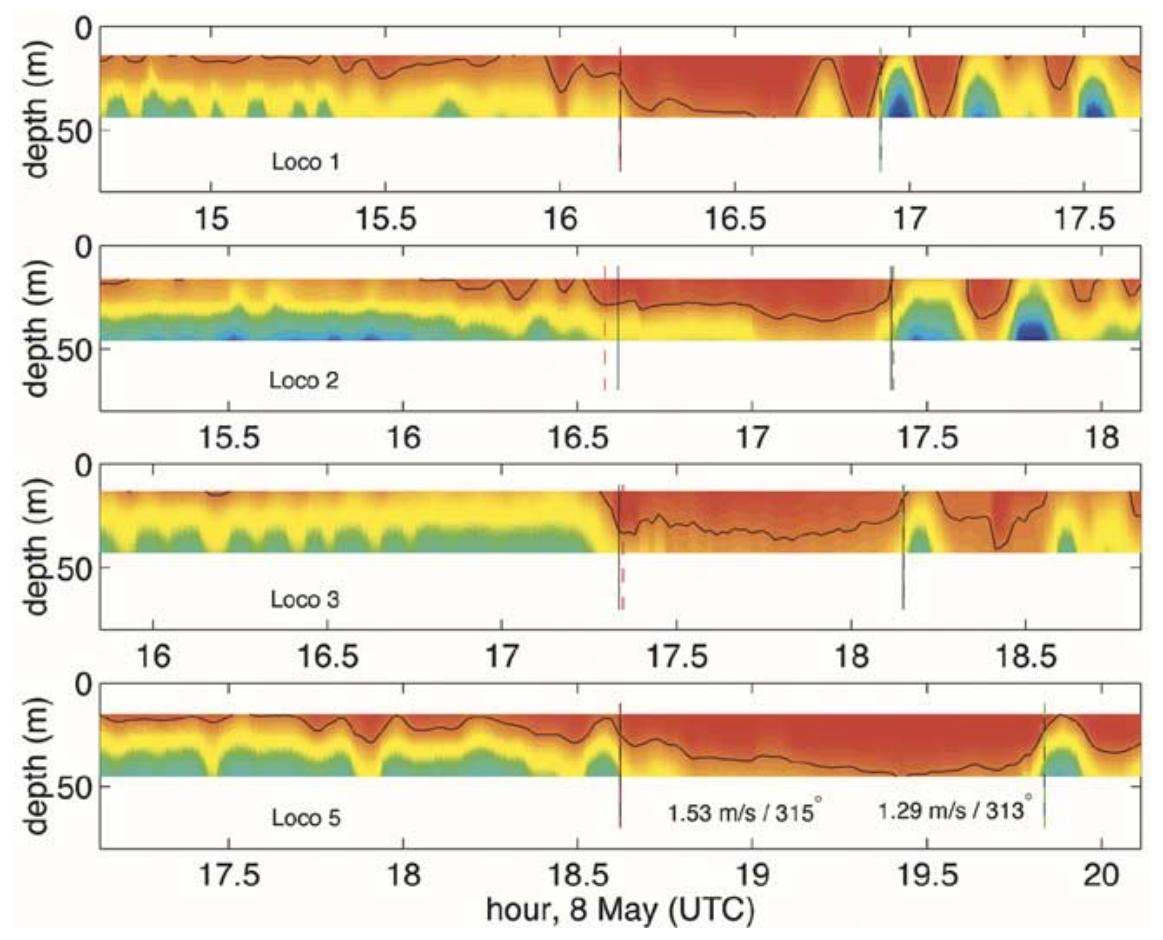

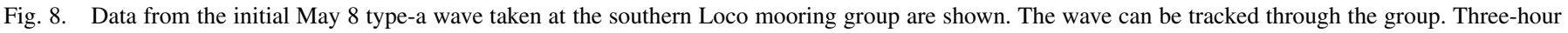

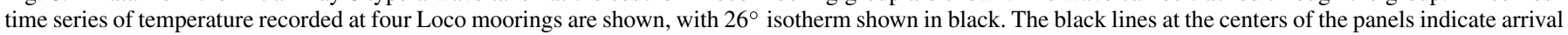

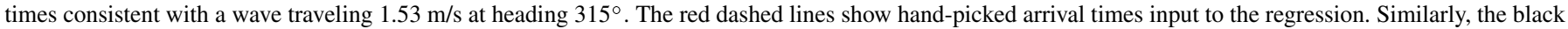
lines in the later parts of the records indicate times consistent with an elevation wave moving $1.29 \mathrm{~m} / \mathrm{s}$ at heading $313^{\circ}$, fit to the times marked with green lines.

sage cannot be verified. The second wave may have existed 4.5 $\mathrm{h}$ before it arrived at S5, with a crest that terminated to the north of S7. Satellite images [20] show such two-dimensional (2-D) wave-crest behavior. At $\mathrm{S} 4$, the front face of the first wave has become shallower in slope than the rear face, giving a skewed wave shape, but the second wave has not changed in this way. The change to skewed shape during shoaling has been previously described and explained [11], [24] and was also observed by ship in ASIAEX [6]. The skewed shapes are very much like those produced by direct numerical simulation [11].

The direction of the upper current in the wave rotates as the waves pass $\mathrm{S} 4$, changing from $280^{\circ}$ to $320^{\circ}$ in the first wave and from $320^{\circ}$ to $340^{\circ}$ in the second wave. The direction rotates less over time if the tidal frequency baroclinic currents (internal tides) are included (i.e., the high-pass filter is not used).

Estimating wave speed from S5 to S4 is simple if it is assumed that the wave travels in the mooring line direction of $340^{\circ}$. The result (for either wave) is $0.95 \mathrm{~m} / \mathrm{s}$. Subtracting the tidal current integrated over this time, $0.13 \mathrm{~m} / \mathrm{s}$ at $316^{\circ}$, gives $0.83 \mathrm{~m} / \mathrm{s}$ wave speed. This speed is lower than most of the estimates in Ramp et al. and is roughly one-half the speed predicted by linear and nonlinear wave theory, also given in that paper. This particular wave yielded different speed estimates than most of the other large initial type-a waves of group 2 . Those waves had speeds of $1.2-1.8 \mathrm{~m} / \mathrm{s}$. No unusual strong subtidal currents were measured at S4 and S5a at this time. Assuming a wave direction other than $340^{\circ}$ gives a slower speed. Orr and Mignerey calculated a speed of $1.17 \mathrm{~m} / \mathrm{s}$ for this wave, with no noticeable decrease in speed over time, and found the propagation direction to change from 318 to $328^{\circ}$ as the wave moved from the $260-\mathrm{m}$ to the $110-\mathrm{m}$ isobath east of our mooring line.
The width of the wave at each station can be computed if a speed is assigned to the wave. Using $1.2 \mathrm{~m} / \mathrm{s}$ gives half-widths $L$ of 580, 930, and $1830 \mathrm{~m}$ at the three sites. Orr and Mignerey report a change from 410 to $2000 \mathrm{~m}$, perhaps using a different criterion for width.

\section{B. May 10 Wave}

The large May 10 wave is shown in Fig. 7. The upper current peaks in the wave are directed toward $300^{\circ}$ at S7 and toward $320^{\circ}$ at both S5a and S4. Assuming linear wave crests the wave speeds can be estimated. Dividing time by distance for these two transects gives $1.75 \mathrm{~m} / \mathrm{s}$ between $\mathrm{S} 7-\mathrm{S} 5$ and $1.52 \mathrm{~m} / \mathrm{s}$ between S5-S4. Mean tidal speeds obtained at S5a during the two periods are $13.8-10.4 \mathrm{~cm} / \mathrm{s}$ at 165 and $260^{\circ}$, respectively. The apparent "push" in the mooring line direction from these will be -13.8 and $1.8 \mathrm{~cm} / \mathrm{s}$, respectively. Subtracting the push gives projected wave speeds of 1.89 and $1.51 \mathrm{~m} / \mathrm{s}$. Multiplying by $\cos \left(340^{\circ}-\right.$ $300^{\circ}$ ) yields wave speed estimates of 1.45 and $1.16 \mathrm{~m} / \mathrm{s}$ in the wave direction $300^{\circ}$. These speeds agree better with the speed predictions given in the Ramp et al. paper than do those of the May 8 wave. No explanation is offered for the difference.

Using $1.45 \mathrm{~m} / \mathrm{s}$ as speed at S7, $1.3 \mathrm{~m} / \mathrm{s}$ at S5a, and 1.16 at $\mathrm{S} 4$, the estimated $L$ at the three sites are 420,740 , and $1250 \mathrm{~m}$, respectively.

This particular wave fits the $\mathrm{KdV} \operatorname{sech}^{2}$ shape at S7 slightly better than the dominant waves of the two previous days. It is of lower amplitude than those waves, 100 versus $160 \mathrm{~m}$, while simultaneously having higher peak upper layer current, 1.6 versus $1.2 \mathrm{~m} / \mathrm{s}$. These differences suggest that individual nonlinear waves of differing character may achieve quasi-permanent form 


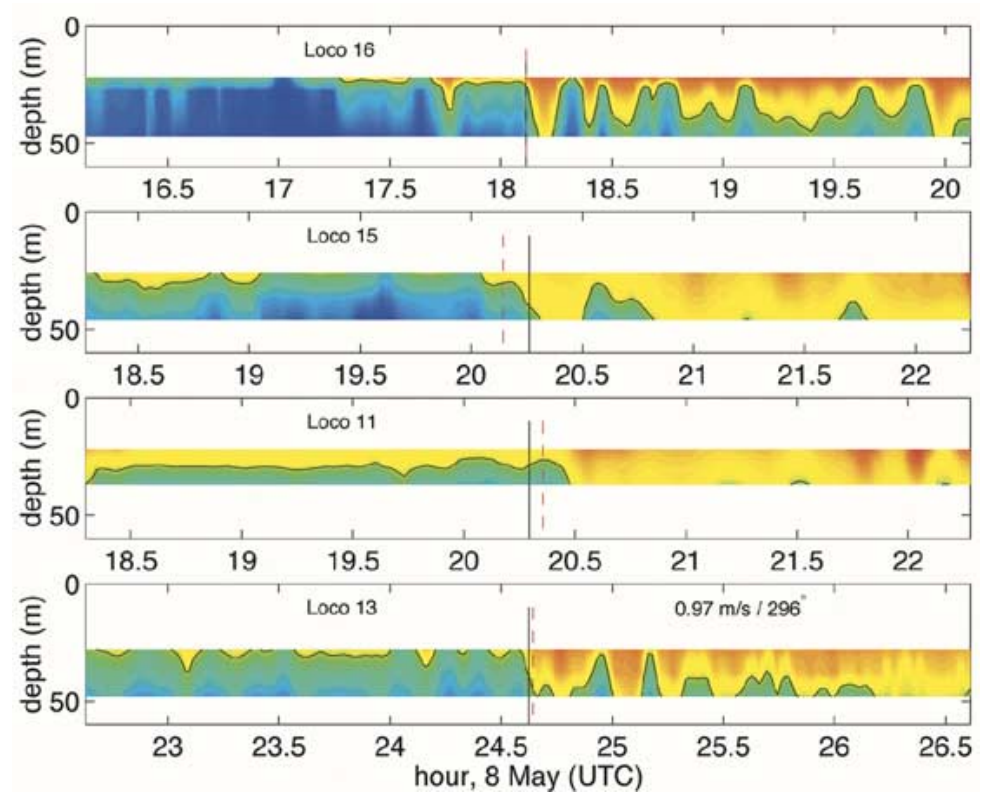

Fig. 9. May 8 wave shown in the previous figure is tracked through the northern Loco mooring group. Temperature is shown. The $24^{\circ}$ isotherm is in black. The black lines show arrival times for a wave moving $0.97 \mathrm{~m} / \mathrm{s}$ at heading $297^{\circ}$, fitted to the hand-picked times shown in red.

through slightly different dynamic balances, although this is only speculation.

\section{May 8 Wave in Shallow Water}

It is occasionally possible to track the evolving transbasin waves into shallow water. The May 8 wave just shown was clearly evident in Loco mooring records, but the May 10 wave was not. Three hours of May 8 temperatures and isotherm heights from four of the southern group Loco moorings are shown in Fig. 8. The figure also shows results obtained by fitting progressive linear wave crests to the May 8 wave data. The fit is performed by solving, via least squares, the expression $Q=\mathbf{D} S_{x y}$, where $Q$ is the $n \times 1$ vector of arrival time differences between an index mooring and $n$ other moorings, $\mathbf{D}$ is an $n \times 2$ matrix of east and north distances between the index mooring and the other moorings, and $S_{x y}$ is a $2 \times 1$ vector containing $x$ and $y$ components of wave slowness (inverse velocity). The solutions are relatively insensitive to small deviations of individual $Q$ values. The leading edge of the depression wave, describable also as an internal bore, arrives at the various moorings at times consistent with a linear crest moving at $1.53 \mathrm{~m} / \mathrm{s}$ with heading $315^{\circ}$. A linear crest also fits the first strong wave of elevation, as shown, moving with speed $1.29 \mathrm{~m} / \mathrm{s}$ at heading $313^{\circ}$. The tidal current integrated over the time period over which the waves move from Loco 1 to Loco 5 is $0.10 \mathrm{~m} / \mathrm{s}$ at $13^{\circ}$. Subtracting this gives resultant feature velocities of $1.48 \mathrm{~m} / \mathrm{s}$ at $313^{\circ}$ and $1.24 \mathrm{~m} / \mathrm{s}$ at $309^{\circ}$. These velocities are close to what is expected for the nonlinear waves in slightly deeper water (see Ramp et al.). It is interesting that these velocities exceed that obtained from the S5a to S4 traversal of this wave.

Fig. 9 shows temperatures from four northern Loco moorings. It is easy to track the front edge of the May 8 wave (or bore) through this group. A fitted linear wave crest moving at $0.97 \mathrm{~m} / \mathrm{s}$ at heading $297^{\circ}$ is shown. The elevation waves are seen to continue northward to these moorings, but are incoherent, so that individual waves cannot be tracked across the order 3-km gaps. a) 200-m site $\mathrm{N}$

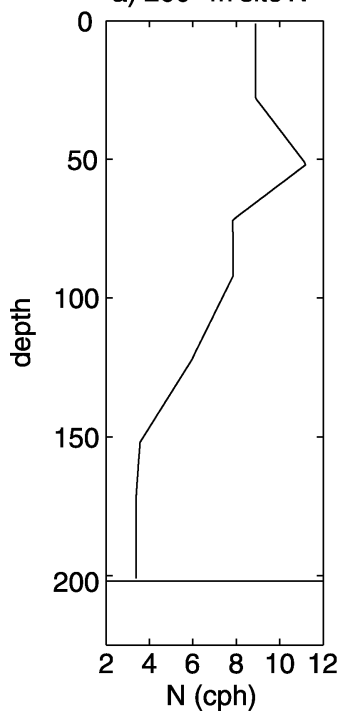

b) Mode 1 displ.

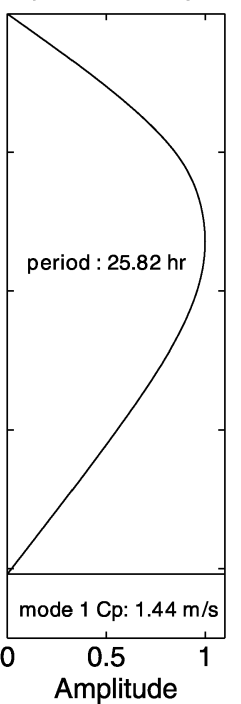

c) Mode 1 current

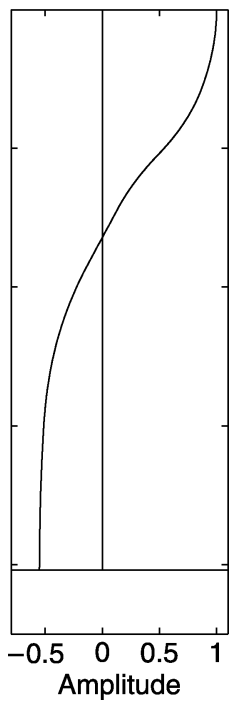

Fig. 10. Stratification and mode-one baroclinic functions at the 200-m depth mooring site. (a) The profile of stability frequency $N$. The profile at the $350-\mathrm{m}$ site is similar at these depths and extends downward with essentially constant $N$. (b) The $\mathrm{O} 1$ tidal frequency mode-one displacement profile. The associated mode-one phase speed is $1.44 \mathrm{~m} / \mathrm{s}$. The speed is $2.11 \mathrm{~m} / \mathrm{s}$ at the $350-\mathrm{m}$ site and is $1.04 \mathrm{~m} / \mathrm{s}$ at the $120-\mathrm{m}$ site. (c) The $\mathrm{O} 1$ mode-one current profile. The zero-crossing moves to $135 \mathrm{~m}$ at the $350-\mathrm{m}$ site, $55 \mathrm{~m}$ at the $120-\mathrm{m}$ site.

This suggests that dissipation and nonlinear processes prevent waves from attaining permanent form and that individual waves appear and disappear on the short time scale of a few hours.

The high-frequency upper layer currents had heading $300^{\circ}$ at both $\mathrm{S} 7$ and S4, suggesting that to be the wave direction (although the currents pointed toward $340^{\circ}$ at S5), so only a slight deflection to the right is seen at the South Loco group. The small incidence angles between the incoming waves and a line normal to the local isobaths (perhaps at $340^{\circ}$ in the mean), plus the large wave amplitudes observed in ASIAEX, may mean that our data set is not appropriate for testing the prediction [10] that small 

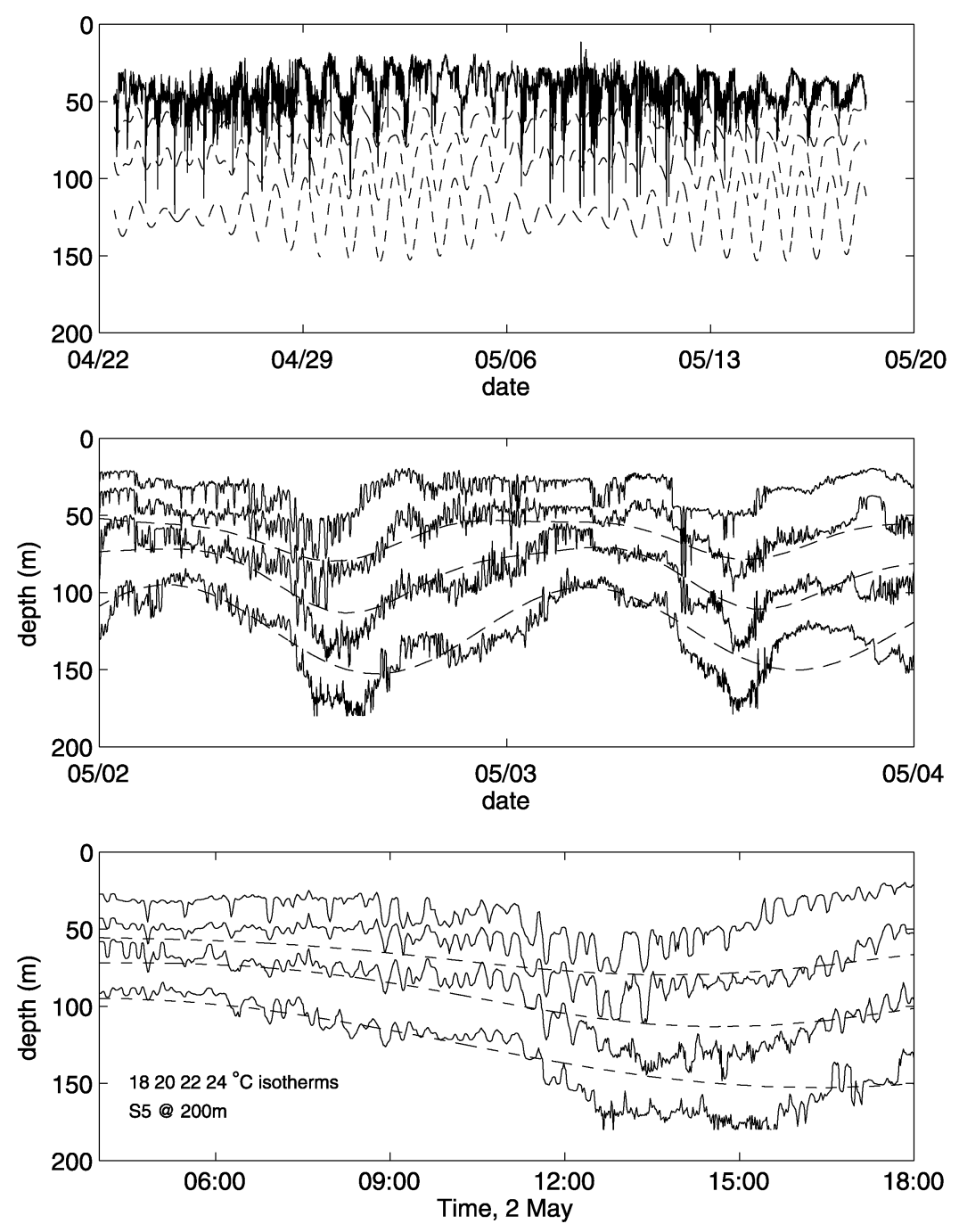

Fig. 11. Isotherm heights measured at $\mathrm{S} 5$ and tidal isotherm displacements derived using harmonic analysis are shown. The top panel shows the $24^{\circ}$ isotherm (solid line) and tidal $22^{\circ}, 20^{\circ}$, and $18^{\circ}$ isotherms (dashed lines) for the duration of the deployment. The middle panel is an expanded view of the same data for a 2-d period having a strong diurnal nature. The unfitted $22^{\circ}, 20^{\circ}$, and $18^{\circ}$ isotherms are added (solid lines). The lower panel shows a further expansion of May 2. The leading edge of the arriving tidal depression has the most high-frequency nonlinear internal wave activity.

amplitude solitary waves will refract to become more oblique to the shelfbreak on the shelf than in deep water. Such refraction would give shallow-water wave headings more westerly than the incident. Despite the mismatch between data and theory, we can report here that such refraction was not observed. Note that the depression wave is very wide in the South Loco area and the front edge may be better described as a bore, moving at a speed substantially higher than the trailing elevation wave.

\section{ShoAling Nonlinear WaVe Discussion}

\section{A. Instability}

The isotherm contours at the 350-m S7 site (Fig. 5) show that the trailing halves of the May 8 and 9 waves are more weakly stratified than the leading halves, indicating mixing. Mixing is also suggested by temperature inversions in the individual sensor time series. Overturning billows within the May 8 wave, likely due to shear instability, were clearly observed by Orr and Mignerey in shallower water and these S7 observations are evidence of earlier dissipation and mixing in deeper water. The process of shear instability within the high-shear zones of these types of waves, and its effect on wave dynamics, has been the subject of previous work [23].

Further evidence of instability in the May 8 wave can be seen at S4. Here, the wave has a skewed shape. Orr and Mignerey observed overturning billows on the shallow-sloped forward face of this wave and the fluctuating isotherm depths along this face, seen in Fig. 6 at times 8.61 to 8.63, lower panels, also indicate this process, although details of the overturns are not resolved by the moored sensors. The forward faces of waves such as these are smooth in numerical simulations [11], but those authors note that their model has subgrid viscosity and diffusivity parameterization and cannot include shear instability. The simulated waves have extended areas of gradient Richardson number less than one quarter, which would be subject to shear instability if it was allowed by the model.

A criterion for a second type of wave breaking has recently been suggested based on numerical simulation of large nonlinear internal waves incident on a slope ([11] and work cited therein). The criterion is that the scaled amplitude $\bar{a}$ exceed $0.8^{\circ} \gamma^{-1}+0.4$, 

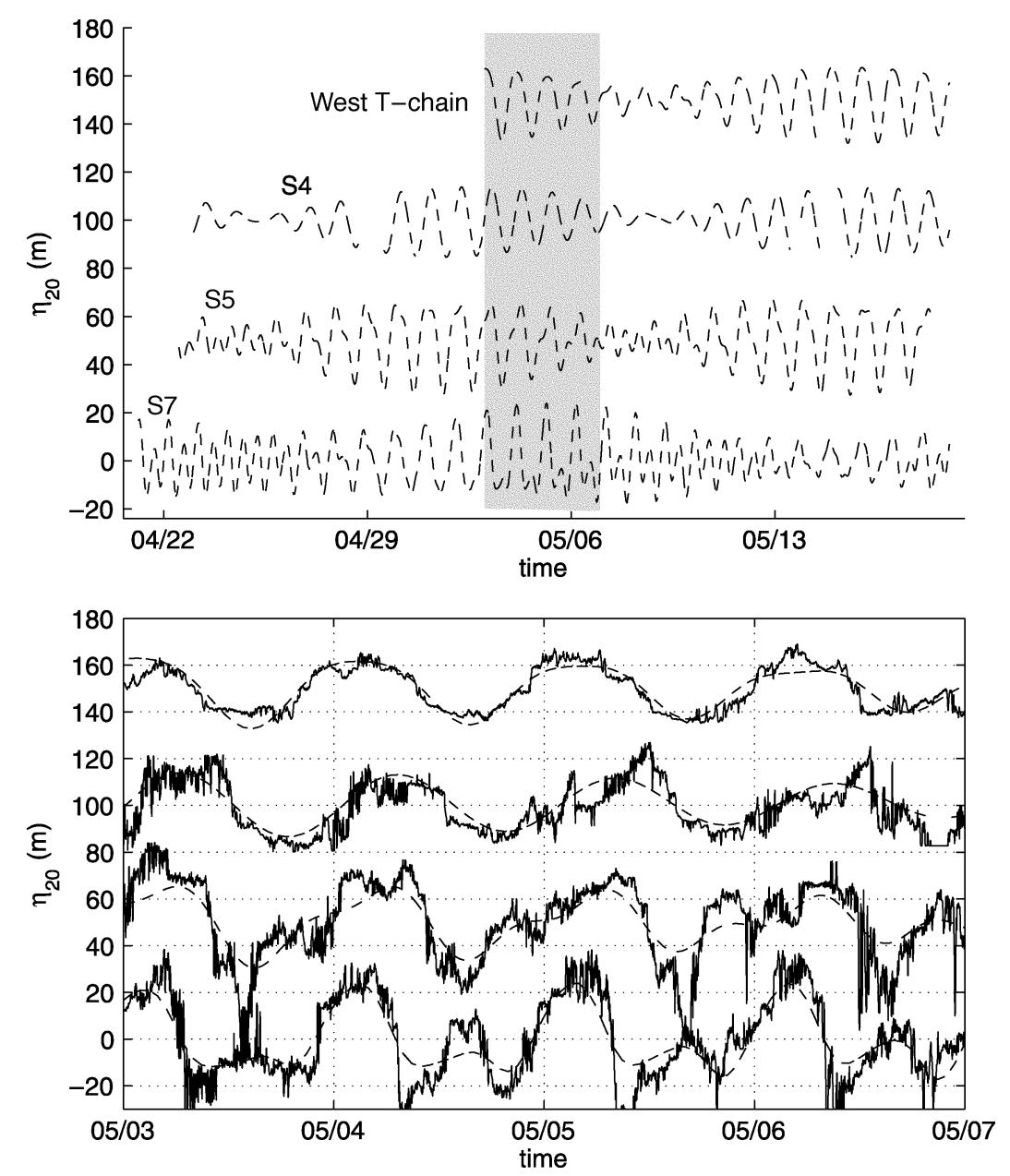

Fig. 12. (Top) Tidally fitted timeseries of $20^{\circ}$ isotherm heights for four sites. Moving from the bottom up, the sites are S7, S5, S4, and the more-western of the two thermometer moorings to the east of the main mooring line. The mean height of each time series is zero, but the series are offset in 50-m increments. (Bottom) An expanded view of $4 \mathrm{~d}$ of the same times series. The time period shown here is highlighted in the upper panel. In addition to the dashed lines repeated from the top panel, the isotherm time series to which tides are fit are shown.

where $\gamma$ is the bottom slope (in degrees) from the horizontal, $\bar{a}=a_{m} /\left(H_{b}-H_{m}\right), a_{m}$ is the maximum internal depression displacement, $H_{b}$ is the water depth, and $H_{m}$ is the upper layer thickness. If this criterion is met, then the rear face of the wave should have an advective (kinematic) instability, with the rear face essentially collapsing into the wave trough. For the two large waves shown in Fig. $5, \bar{a} \approx 0.48$. The bottom slope at this site is only on the order of $0.3^{\circ}$ (a chart of bottom slope appears in a later section), so the criterion is not satisfied; $\bar{a}$ in excess of 2 would be required. It is also not satisfied in the $20-\mathrm{km}$ section traversed by Orr and Mignerey, where the slope was uniformly near $0.4^{\circ}$. The fact that the breaking criterion is not satisfied in the Orr and Mignerey transect, nor at the 350-m and 200- and 120 -m sites (where $\bar{a}$ increases only to 0.65 , but the slopes do not increase) is consistent with the overall stability (lack of catastrophic collapse) that we observe in the rear face of these waves. The observed large waves of depression continue to be recognizable although dissipative, even as elevation waves are created behind them. The rapid dissipation of wave energy observed by Orr and Mignerey and the transfer of energy in trailing waves as discussed in the next section, suggest that the wave amplitude may decrease steadily as the waves shoal, preventing the attainment of the kinematic instability breaking criterion.

\section{B. Energy Flux}

The behavior of a large nonlinear depression wave at the slope can be understood using the expression for energy flux due to pressure work $\mathbf{F}=\mathbf{u}^{\prime} p^{\prime}$, where $\mathbf{u}^{\prime}$ and $p^{\prime}$ are wave velocity and pressure and where $\mathbf{u}^{\prime}$ is evaluated in a reference frame moving with the wave. Flux due to advection of kinetic energy is neglected in this discussion [25]. F can be recognized as the force per unit area times velocity, which is force times distance (equal to work or energy) per unit time per unit area. $\mathbf{F}$ has units $\mathrm{W} / \mathrm{m}^{2}$. Integration over depth for linear crested waves gives the flux per unit length of wave crest, with units W/m. For a system with a thin upper layer and a thicker lower layer, much of the "action" regarding work and flux for a mode-one wave in this frame is in the lower layer, where $\left|p^{\prime}\right|$ has high values, as does $\mathbf{u}^{\prime}$.

Limiting ourselves to horizontal flux through vertical planar surfaces normal to the direction of a wave, so that only the normal component $u^{\prime}$ of $\mathbf{u}^{\prime}$ is relevant, we have $F=u^{\prime} p^{\prime}$. Within waves of permanent form the primary flux is forward, in the direction that the wave is moving, and is concentrated in the lower layer underneath the depressed thermocline. Here, $u^{\prime}$ is large and negative (opposite the direction the wave is moving) and $p^{\prime}$ is also negative, giving positive $F$. Perturbation $p^{\prime}$ is negative because less-dense surface water occupies an increased 
(a) $350 \mathrm{~m}$ site : $42.5 \mathrm{~m}$ level : $41 \%$ var. fit

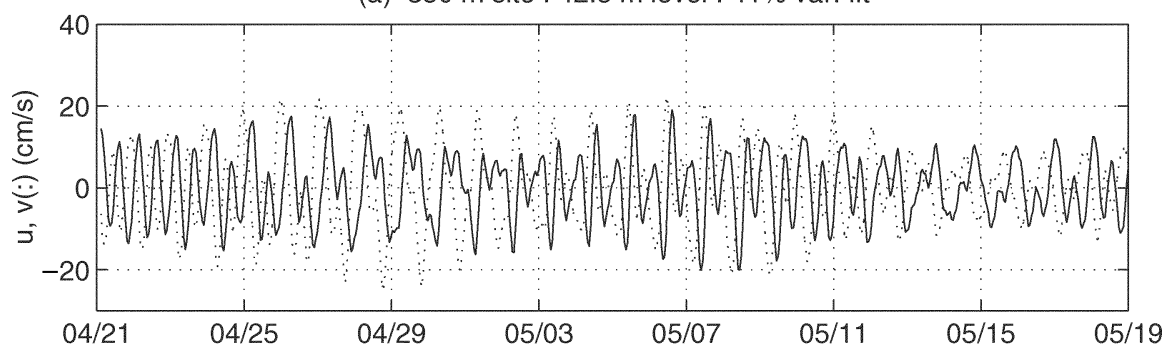

(b) $200 \mathrm{~m}$ site : $45 \mathrm{~m}$ level : $56 \%$ var. fit

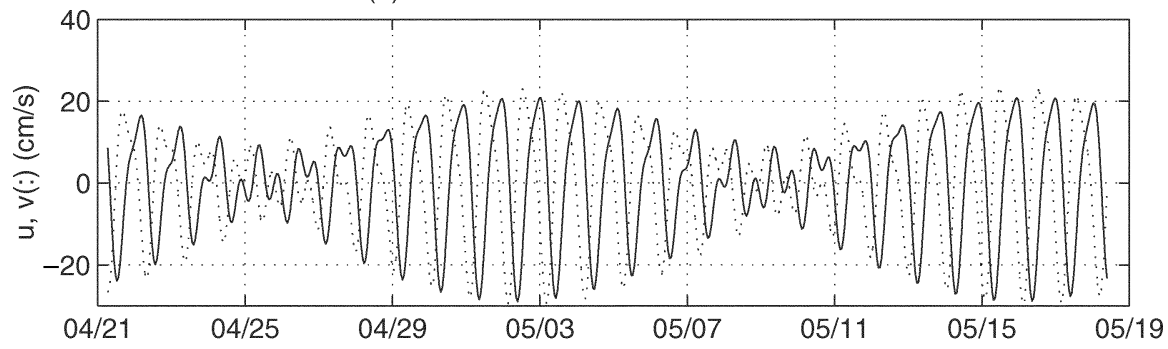

(c) $120 \mathrm{~m}$ site : $30.5 \mathrm{~m}$ level : $55 \%$ var. fit

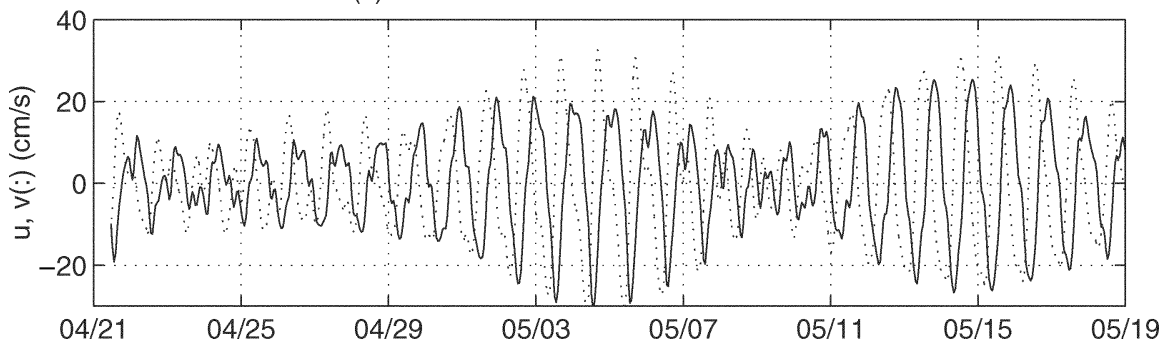

(d) Barotropic tide $V$ at S5a; offset S7 p x $4(\mathrm{db})$

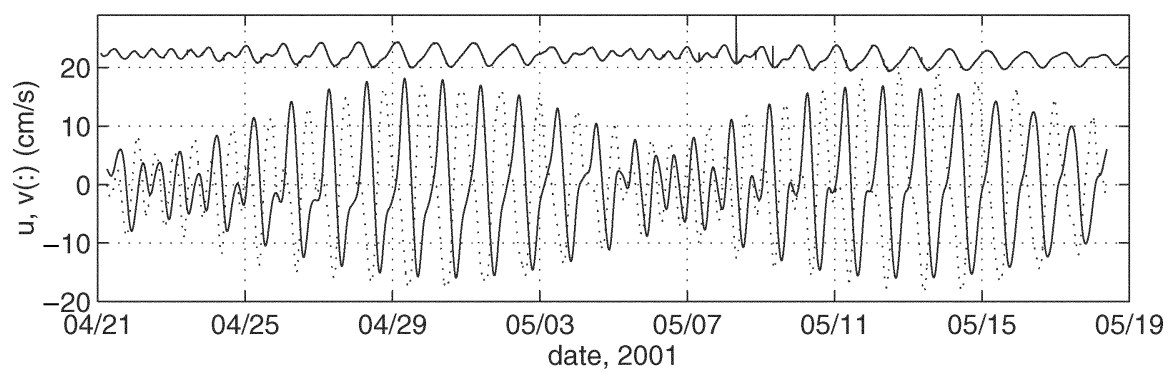

Fig. 13. Fitted upper-level tidal time series at three moorings sites are shown. (a) Time series for S7. The line shows the eastward component $u$, the dashed line shows the northward component $v$. (b) Time series for S5a, to the north of S7. (c) Time series for S4, to the north of S5a. (d) The record of bottom pressure (surface tide) from S7 site is shown at the top. Units are decibels, with scale factor of 4 applied to the data. Also shown is the S5a barotropic current time series. All current time series have predominantly clock-wise (anticyclonic) rotation.

fraction of the water column. The negative $p^{\prime}$ accelerates the flow under the wave and is consistent with the increased speed. The forward flux serves to pull water underneath the wave, sustaining the entire wave and propelling it forward. There is a smaller backward flux in the upper layer, which decelerates water that has passed under the depression.

As the lower layer thins under shoaling conditions, the flow under the wave is disrupted because of bottom drag and possible shear instability at the thermocline in the leading edge of the wave. Orr and Mignerey have observed such instability [6] and it has also been predicted using numerical simulations [11]. The energy flux could be sustained during the shoaling process if the thinning of the lower layer were to be compensated by increased flow speed, but drag and internal instability prevent this.

Because the forward energy flux at the base of the wave is thus restricted, some of the wave energy must flow backward out of the wave or the wave must grow in strength (energy density). The instability at the interface shows that the energy density can grow no more; thus, the energy must flow backward. The manner in which the backward energy flux manifests itself can also be understood in terms of $u^{\prime} p^{\prime}$. In the zone in which the upper layer is depressed below its undisturbed depth, the flow is positive $\left(u^{\prime}>0\right)$ but $p^{\prime}$ is negative, as in the lower layer. This gives the backward energy flux that removes energy from the wave. This flux exists prior to shoaling, but as flow is diminished in the lower layer it exceeds the lower layer forward flux in magnitude.

The horizontal scale of the waves increases as they shoal, one response to the negative flux. Another consequence of the backward flux is the upward and forward movement of the lower layer behind the wave. This creates the elevation wave immediately behind the (now distorted) original depression wave. The 
(a) 350-m site

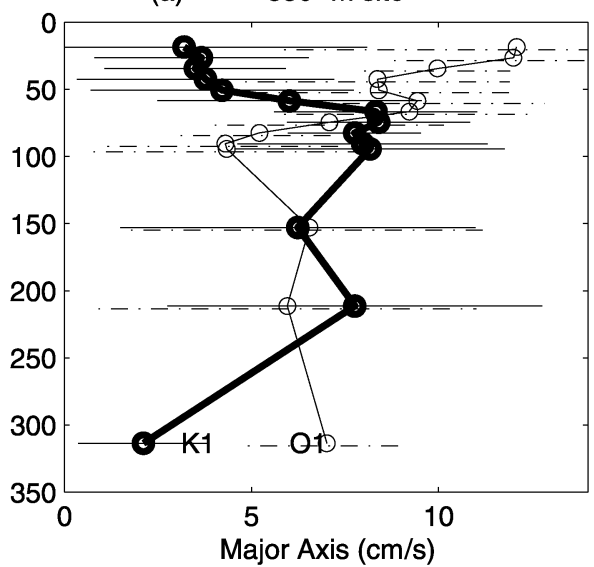

(c)

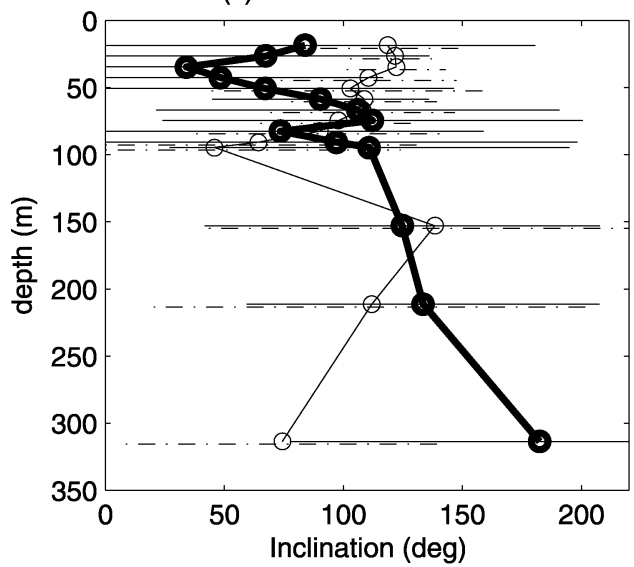

(e)

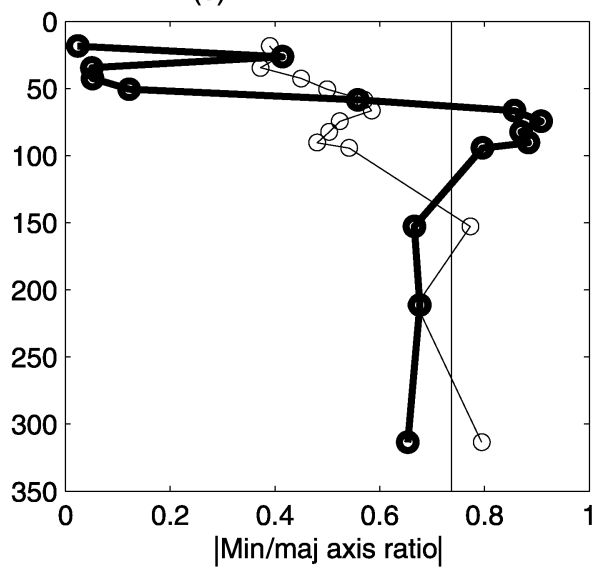

(b)

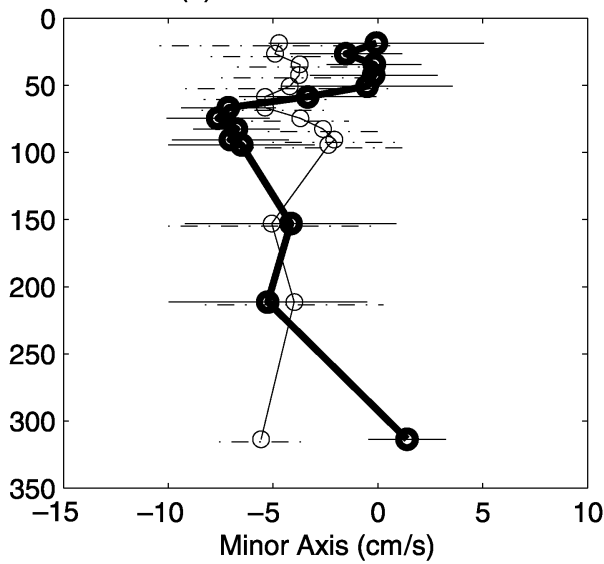

(d)

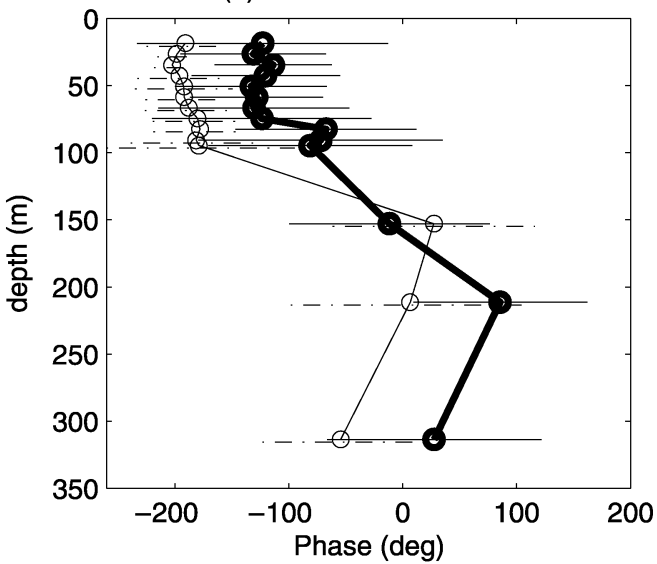

(f)

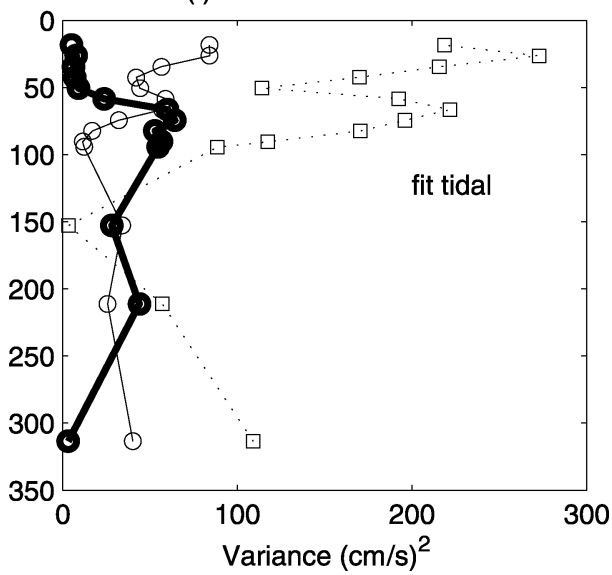

Fig. 14. Parameters at two diurnal tidal frequencies obtained by tidal analysis at multiple depths of the 350-m site baroclinic current vectors. Thick lines: K1. Thin lines: O1. (a) Major axis amplitude. (b) Minor axis amplitude. Negative amplitude indicates clock-wise current rotation. (c) Current ellipse inclination anticlock-wise from east. (d) Phase with respect to astronomical forcing. Increasing phase with depth means that the tide is delayed with depth, so the phase velocity is downward. (e) The absolute value of the ratio of major to minor axis lengths. The vertical line shows the expected ratio $\omega / f$ for internal waves of (diurnal tidal) frequency $\omega$. (f) Variances of each of the fitted tidal constituents, with the total variance of the fitted tidal time series at each depth also shown.

elevation wave is efficiently able to flux energy forward because it has positive $u^{\prime}$ in an extensive lower layer where $p^{\prime}$ is positive and of high magnitude.

Because of domed isopycnals and rearward flow, there is rearward energy flux within the elevation wave in the lower part of the upper layer. This leads to the generation of another wave to the rear, and so on. The balance of the initial wave of permanent form is broken by the sloping bottom and appears in our data to never be regained.

\section{INTERNAL TIDE OBSERVATIONS}

Internal tide signals are readily apparent in unfiltered temperature and velocity data (Figs. 3 and 4). The nonlinear internal waves shown in many of the figures appear to be well approximated by mode-one solutions in the vertical, but there are indications that the internal tides behave as beams and travel along sloped internal wave characteristics. Fig. 4 clearly shows a diurnal oscillation that progresses downward with 
(a) 200-m site

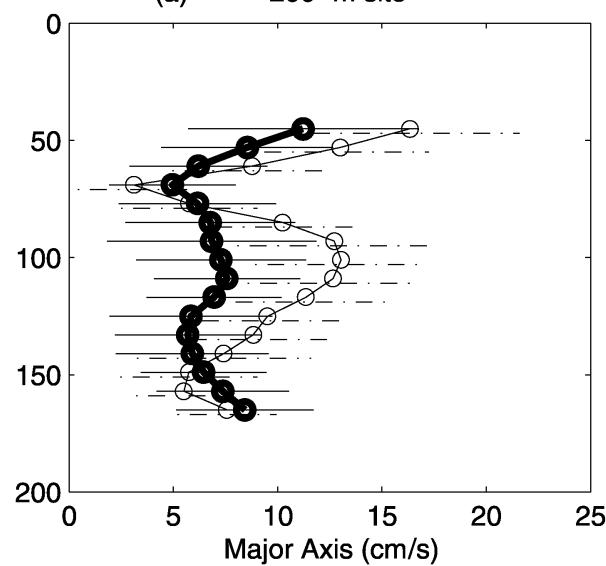

(c)

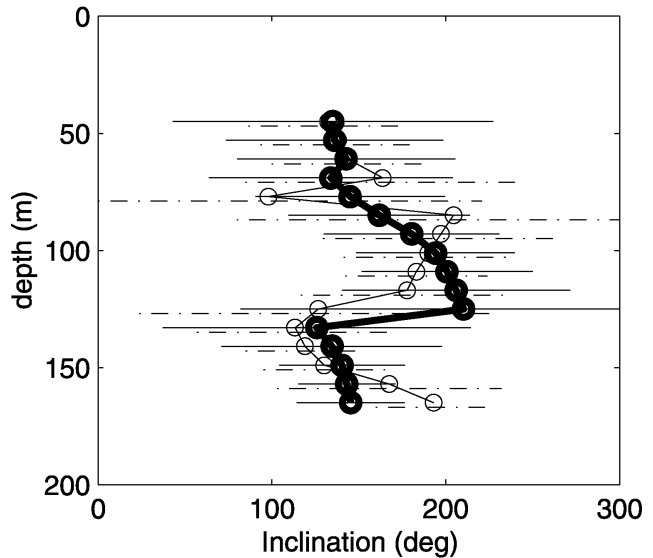

(e)

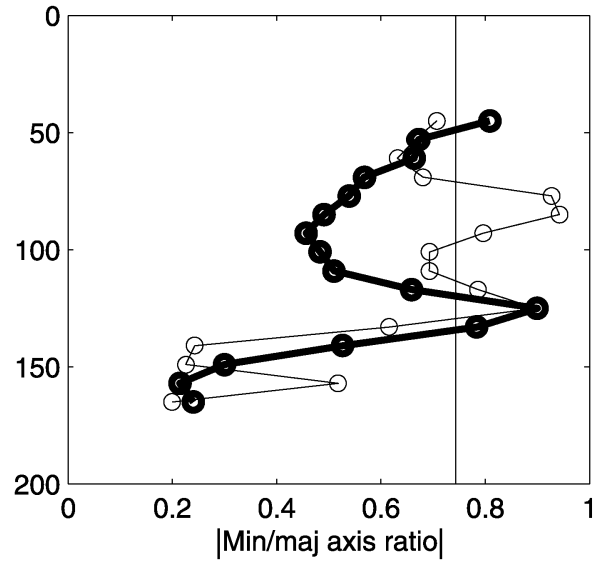

(b)

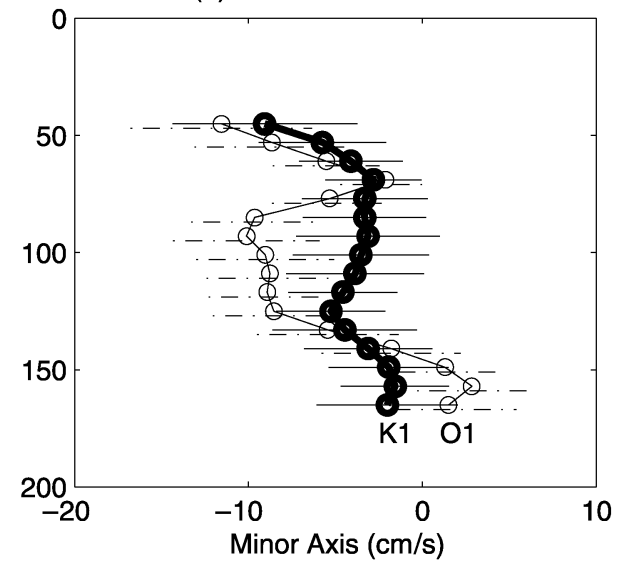

(d)

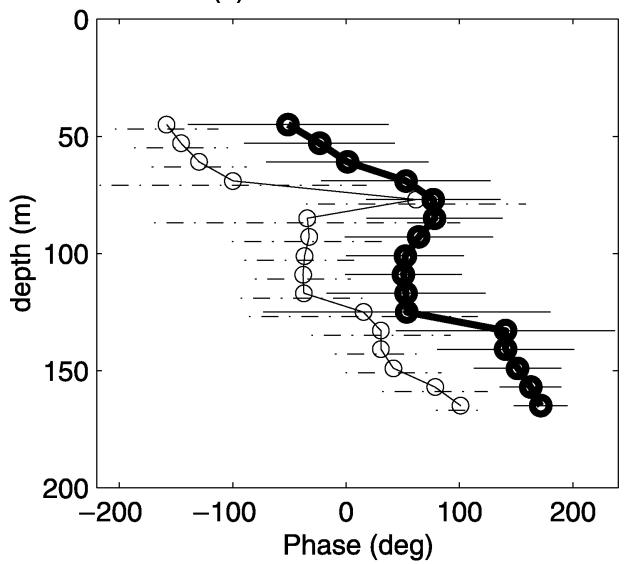

(f)

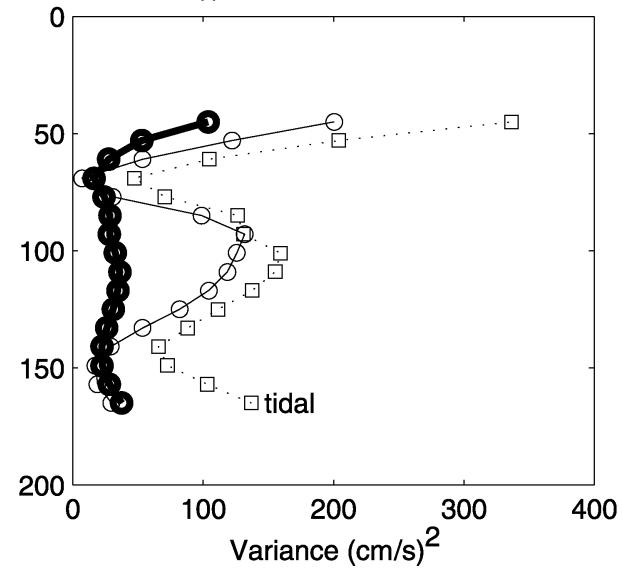

Fig. 15. Parameters at $\mathrm{O} 1$ and K1 frequencies obtained by tidal analysis at multiple depths of the 200-m site (S5a) baroclinic current vectors. Data are presented as in the previous figure.

time. Despite the fact that the internal tides thusly depart from a modal structure, some modal properties of the local baroclinic waveguide are presented here for comparison with the data. The mean buoyancy frequency profile $N(z)$ measured at S5 is shown in Fig. 10. The mode-one functions for displacement and horizontal current at the $\mathrm{O} 1$ principal lunar diurnal tidal constituent are also shown [26]. The exercise of computing mode functions at the various moorings sites provides horizontal phase velocities, ranging from $2.1 \mathrm{~m} / \mathrm{s}$ at $\mathrm{S} 7$ to $1.0 \mathrm{~m} / \mathrm{s}$ at S4, along with other aspects of the mode functions as given in the figure caption.
Under the premise that internal waves are generated by interaction of barotropic tidal currents with a sloping bottom [27]-[29] and because of the observed downward phase propagation with time, it is sensible to consider internal waves of tidal frequency that are not mode-like. Internal wave kinematics are linked to the quantity $C=\left(\omega^{2}-f^{2}\right)^{1 / 2} /\left(N^{2}-\omega^{2}\right)^{1 / 2}$, where $f$ is the Coriolis parameter and $\omega$ is the internal wave frequency [26]. This quantity is equal to $\left|k_{h} / m\right|$, the ratio of horizontal to vertical wavenumbers, and is equal to the cotangent of the angle that the wavenumber vector makes with the horizontal. The group velocity vector, tangent to a ray characteristic, is 
(a) $\quad 120-m$ site

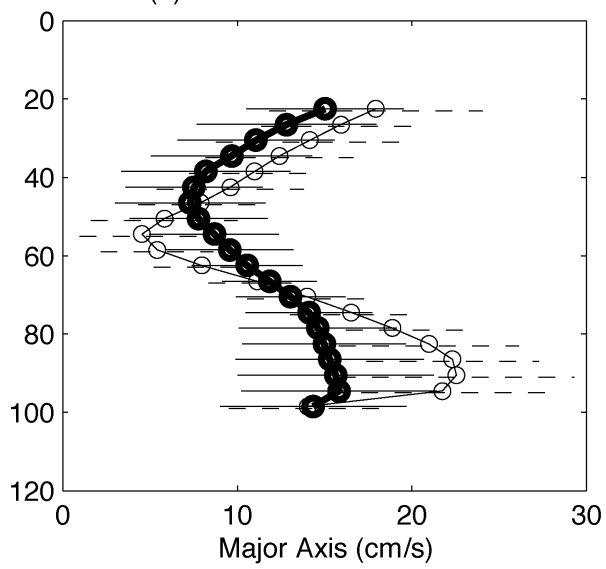

(c)

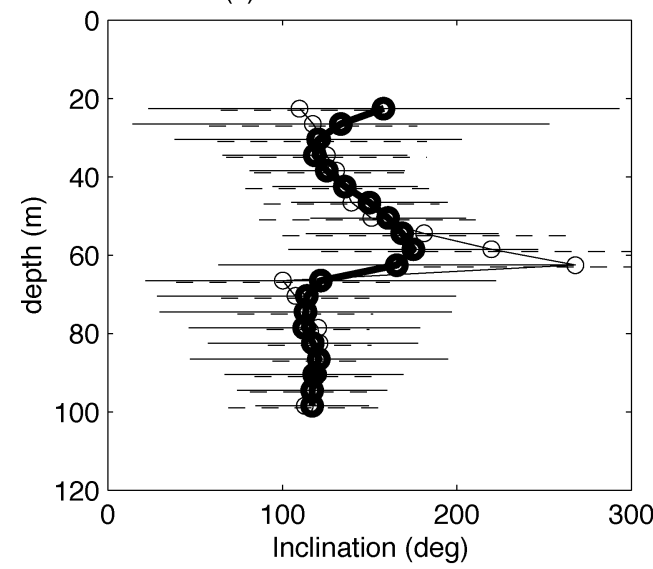

(e)

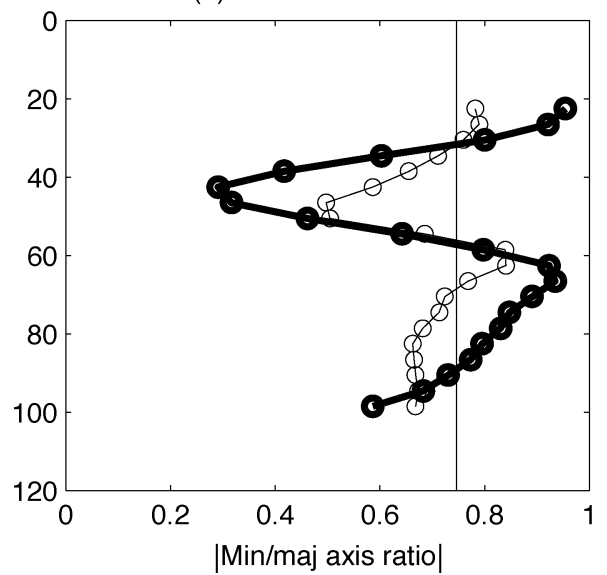

(b)

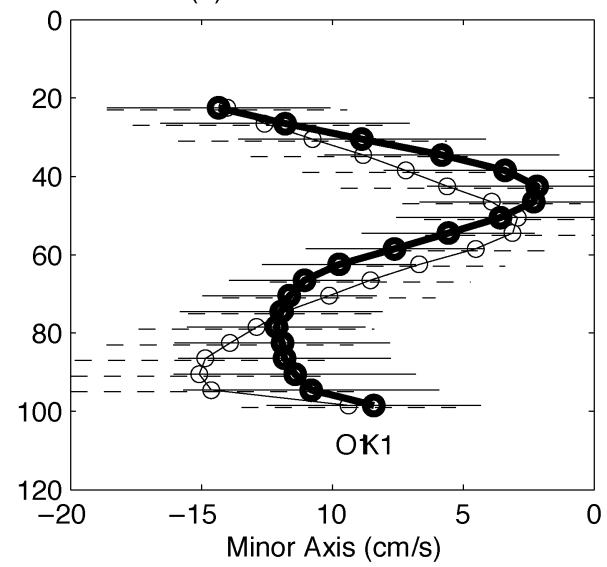

(d)

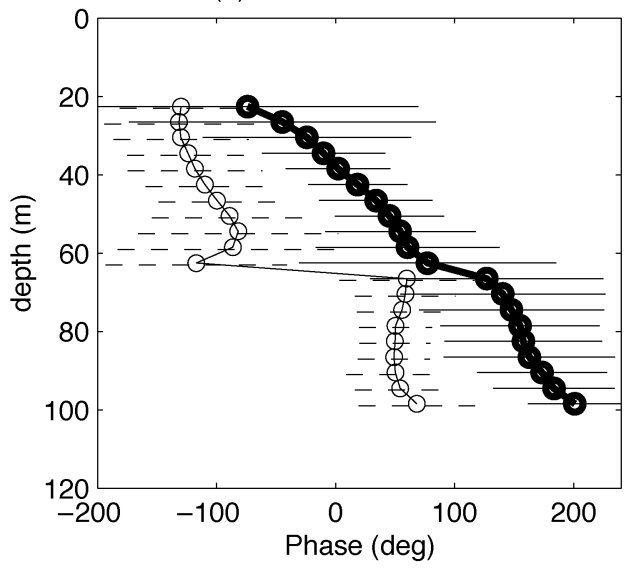

(f)

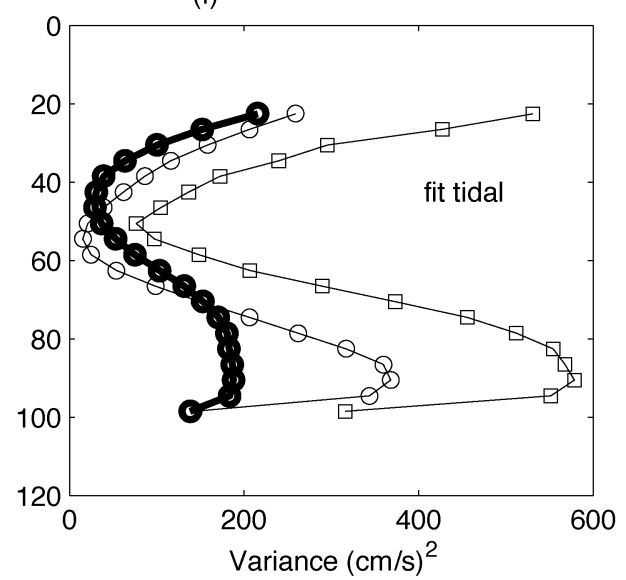

Fig. 16. Parameters at $\mathrm{O} 1$ and $\mathrm{K} 1$ frequencies obtained by tidal analysis at multiple depths of the 120-m site (S4) baroclinic current vectors. Data are presented as in the previous figure.

normal to the wavenumber vector and of opposite vertical sign. The horizontal phase velocity $c_{p}=\omega / k_{h}$ is easily computed and is a linear function of either horizontal or vertical wavelength if $N$ is constant. Example values of $c_{p}$ for O1-frequency internal waves range from $1.0 \mathrm{~m} / \mathrm{s}$ for waves of $350-\mathrm{m}$ vertical wavelength and 90-km horizontal wavelength to $0.5 \mathrm{~m} / \mathrm{s}$ for waves of $175-\mathrm{m}$ vertical wavelength and $46-\mathrm{km}$ horizontal wavelength, each computed for $N=6$ cycles per hour.

Internal tidal isotherm displacements at one site, isolated from the total isotherm time series using harmonic analysis, are shown in Fig. 11, where they are compared directly with the total time series. Tidal analysis was performed on low-pass filtered data converted to selected hourly values using the procedure described previously. The procedure fits roughly $50 \%$ of the variance in each of the tidal-band isotherm time series. Submeter displacements from barotropic tides will be mixed in with the internal tidal signals here, but are much smaller. The signals that do not fit are presumably other internal waves that are not tidal in origin or that are not coherent throughout the month-long record. Tidal-frequency isotherm displacements were successfully computed at every mooring with dense vertical thermometer spacing. Fits at four sites are shown in Fig. 12. 
(a) $\quad 350-m$ site

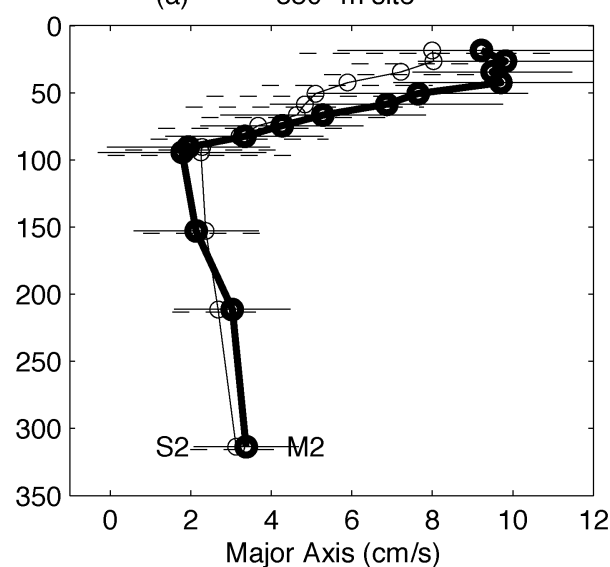

(c)

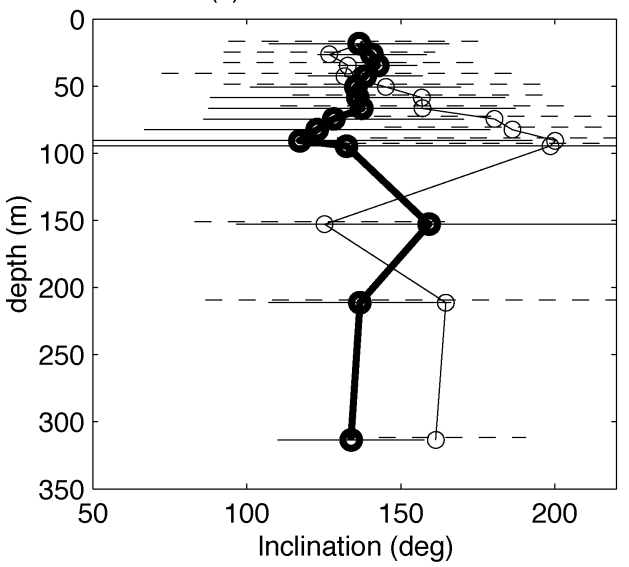

(e)

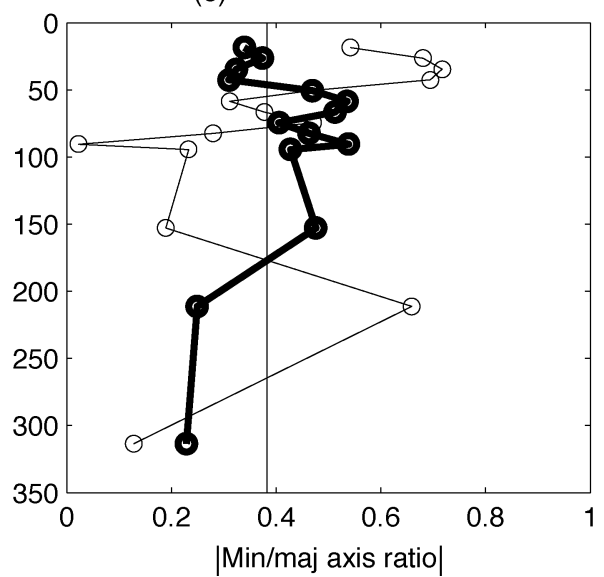

(b)

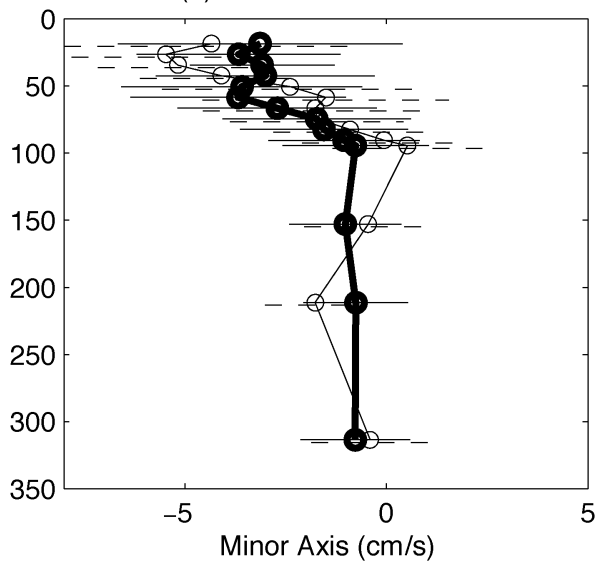

(d)

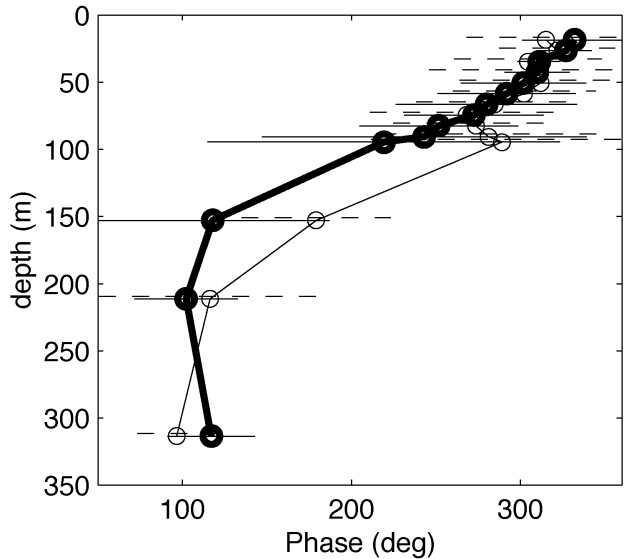

(f)

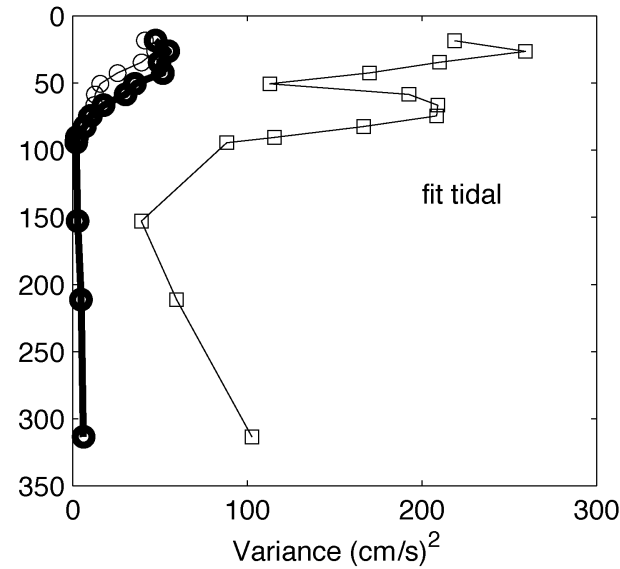

Fig. 17. Parameters at two semidiurnal frequencies (M2 and S2) obtained by tidal analysis at multiple depths of the 350-m site (S7) baroclinic current vectors. Data are presented as in the previous figure. Thin lines: S2. Thick lines: M2.

In addition to fitting of tides to isotherm displacements, internal tidal velocity parameters can be found as functions of depth for each ADCP record. First, the barotropic tidal time series computed from each record was removed [12]. Tidal fitting was then performed on the residual current at each depth. This separates the baroclinic tidal phases and ellipse geometries from their barotropic (esentially depth-independent) counterparts. The barotropic waves travel at 50-200 m/s, much faster than the $1-2 \mathrm{~m} / \mathrm{s}$ phase velocities of the internal waves, but they have current ellipses of the same magnitude as the baroclinic waves, so it is necessary to remove them. The tidally fit portions of upper-depth baroclinic velocities are shown in Fig. 13. The S7 (350-m) tidal height time series is shown at the top of panel d (the trace is offset and multiplied by 4; the maximum tidal excursion is about $1 \mathrm{~m}$ peak-to-peak). The internal tidal currents at S5a and S4 are similar to each other, but differ from the offshore S7 time series. The barotropic tidal currents at S5a, estimated very reliably from the data, are shown in Fig. 13(d). The internal tidal currents are similar to the barotropic current, but their spring/neap cycles lag their barotropic counterparts by a few days. The S7 upper-layer internal tide can be seen to be a complicated sum of diurnal 


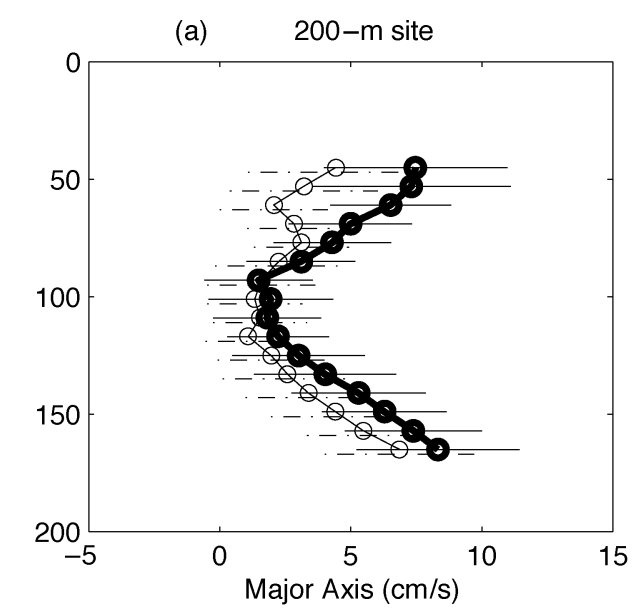

(c)

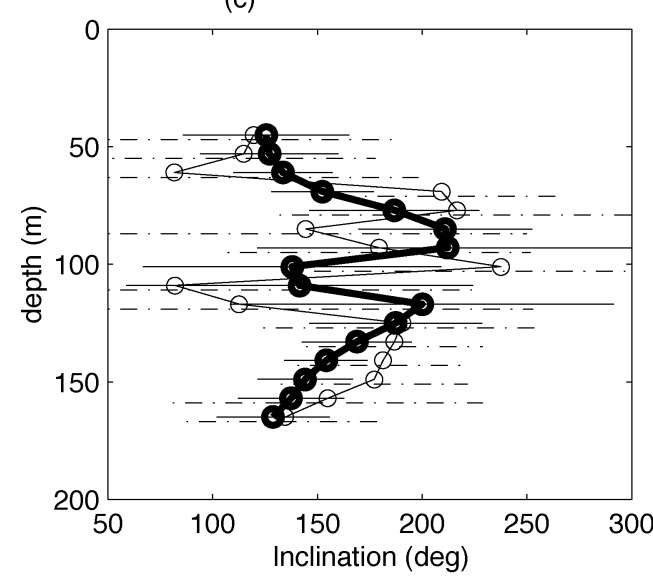

(e)

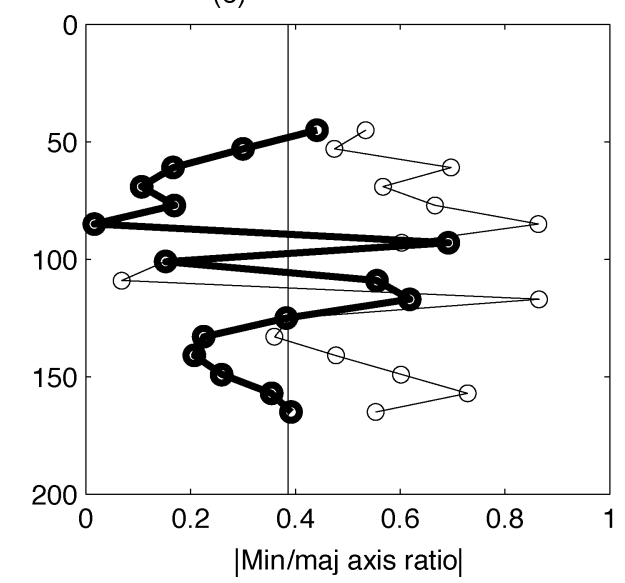

(b)

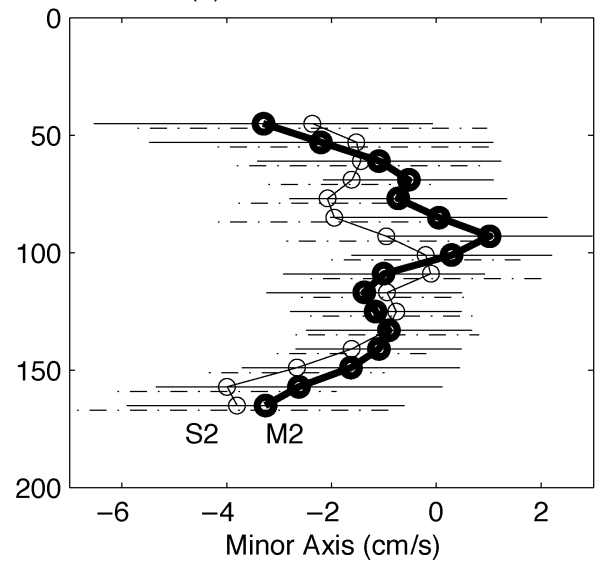

(d)

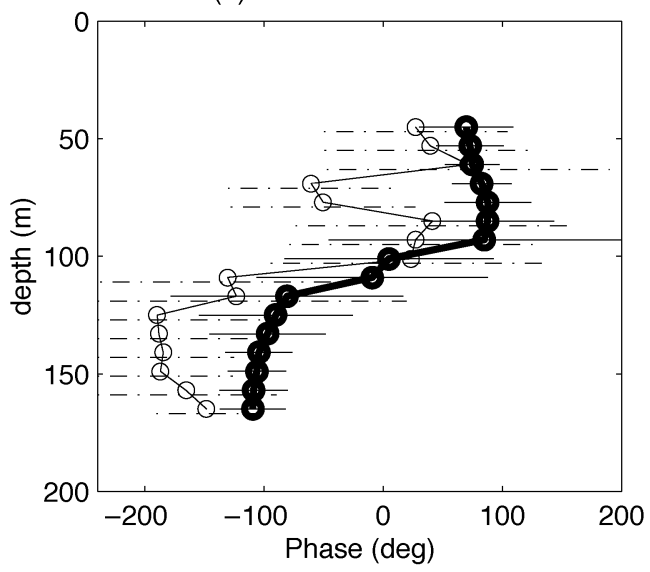

(f)

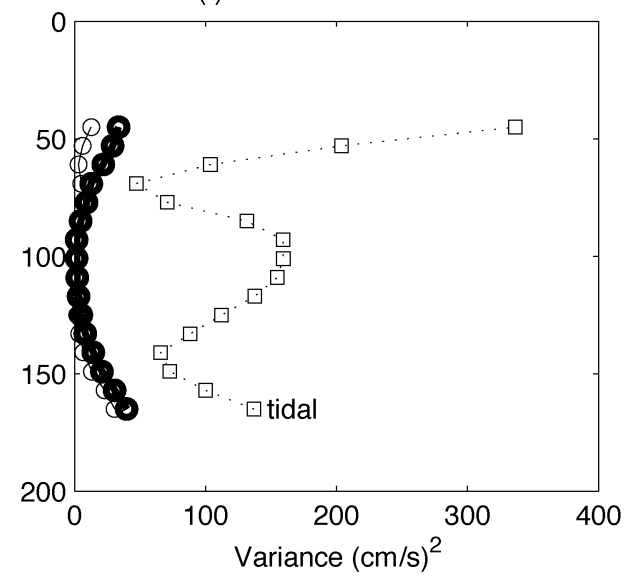

Fig. 18. Parameters at M2 and S2 frequencies obtained by tidal analysis at multiple depths of the 200-m site (S5a) baroclinic current vectors. Data are presented as in the previous figure.

and semidiurnal waves, not resembling the barotropic tide or the other internal tides.

Baroclinic tidal parameters were computed at many depths for the currents recorded at S7, S5a, and S4. At S7, 25 to $62 \%$ of time series variances from various depths were explained by the fit, with a typical result of $55 \%$. At S5a, 22 to $56 \%$ of the variances were explained, with a typical result of $45 \%$. At S4, 31 to $76 \%$ of variances were fit, with a typical result being $65 \%$. At each site most of the baroclinic variance was explained by two diurnal (O1 and K1) and two semidiurnal constituents (M2 and S2), as with the barotropic variance.
Current ellipse parameters for the two dominant diurnal constituents are shown in Figs. 14-16. A negative minor axis indicates clock-wise velocity rotation. Inclination gives the major axis direction measured anticlock-wise from east. For example, a monochromatic linear internal wave traveling to the north would have inclination $90^{\circ}$, and the ratio of its minor to major axes would equal the linear internal wave value of $f / \omega$. Values of $f / \omega$ are plotted as lines in each (e) panel to compare with the axis ratios. The consistent results over the various depths at many sites suggest that the error bars are conservative. 
At each site, the Greenwich phase (panel d) is seen to increase with depth, consistent with an internal wave having downward phase velocity and upward group velocity (cf. [26]). The observed ratios of minor to major axis hover around the predictions but do not fit precisely. The inclinations, which would be aligned with the horizontal wavenumber (propagation direction) of a linear internal wave, are near $120^{\circ}$ at middepth for $\mathrm{S} 7$, are near $140^{\circ}$ at the surface and bottom for S5a (the depths which show consistent phases), and are between 110 and $160^{\circ}$ for S4. These inclinations indicate diurnal wave propagation to the northwest (or southeast). The K1 phases and inclinations for the $\mathrm{S} 4$ position (Fig. 16) show the most consistency, with phase rotating $300^{\circ}$ smoothly with depth. The upper and lower K1 inclinations and phases at S5 (Fig. 15) show the same consistency, with some aberrations in the middle of the water column.

Parameters for the two dominant semidiurnal constituents are shown in Figs. 17 and 18. The upper water column at S7 shows consistent inclinations and phases, with vertical phase progression reversed from the diurnal behavior, indicating downward semidiurnal group velocity. The inclinations at S7 are again in the vicinity of $130^{\circ}\left(320^{\circ} \mathrm{T}\right)$. The minor to major axis ratios are distinctly different from the diurnal values and hover near the expected value of 0.4 . The S5a site has a very low amount of semidiurnal energy, but at a few depths gives phase, inclination and axis ratio values consistent with the $\mathrm{S} 7$ results, suggesting that the same waves are present.

To further compare the baroclinic current fits at the three sites, note that the $\mathrm{O} 1$ tidal constituent is the only significant diurnal constituent fitting near the surface at S7. K1 and NO1 (Doodson number $\left.\begin{array}{llllll}1 & 0 & 0 & 1 & 0 & 0\end{array}\right)$ constituents are also fit at S7 with less reliability, along with $\mathrm{M} 2$ and $\mathrm{S} 2$ of magnitude comparable to $\mathrm{O} 1$ and $\mathrm{K} 1$, resulting in a complicated structure of the fitted time series over the month (Fig. 13). At S5a and S4, the dominant constituents are $\mathrm{O} 1$ and $\mathrm{K} 1$, giving rise to a fortnightly springneap pattern in the fitted series. M2 and S2 constituents of lower magnitude than $\mathrm{O} 1$ and $\mathrm{K} 1$ are also fit at those sites.

Fig. 19 shows the baroclinic tidal ellipses as functions of depth for the 350-m and the 120-m sites. Note how the ellipse sizes, phases, and inclinations are steady with depth. Note also how the diurnal constituents are are larger at the shallower site than at the deep site, while the semidiurnal do not show this behavior.

Table II gives barotropic and baroclinic current tidal ellipse major/minor axis current amplitudes, inclinations, and phases. The baroclinic entries are for representative near-surface depths. The barotropic entries tell us a number of things. The $\mathrm{O} 1$ and $\mathrm{K} 1$ currents tend to be stronger at S4 and S5a than at S7, with consistent inclination to the northwest at S5a and S4. There are lateral tidal height and depth changes, so these numbers do not necessarily indicate mass flux divergence. The M2 and S2 currents are weaker than the diurnal currents at each site, with essentially northerly inclinations at S5a and S4. At S7 the inclinations of all four constituents are best described as westerly. A reasonable hypothesis is that these barotropic currents interact with the sloping bottom to force the baroclinic tides, as described by Baines and others [27].

The baroclinic tidal entries in the table do not precisely mimic the barotropic entries, but there is enough similarity
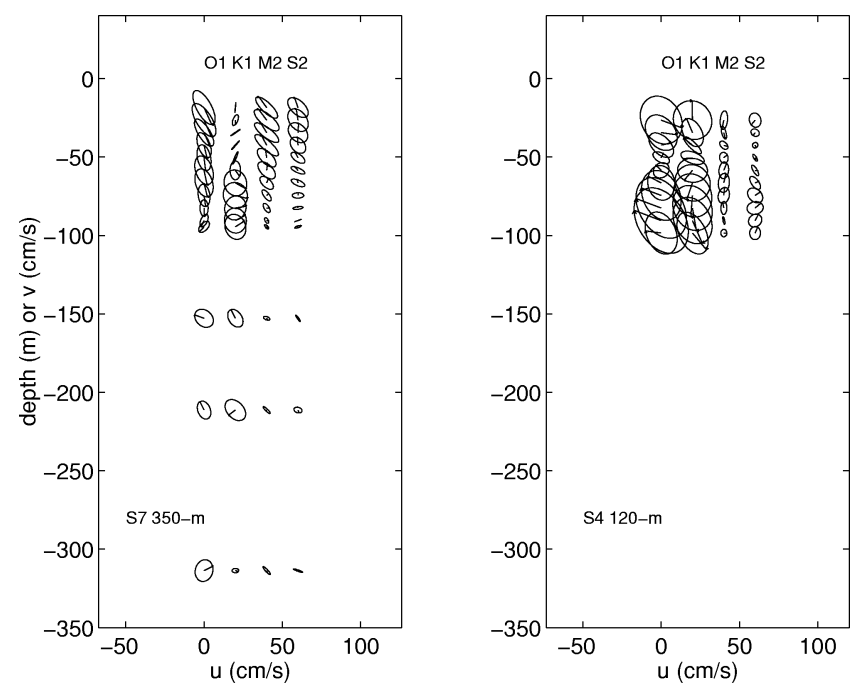

Fig. 19. Tidal ellipses for four constituents are shown for the S7 and S4 sites. The ellipses are centered at the measurement depths. The lines from the center show the Greenwich phases. The major axes point in the directions of the inclinations, with west being to the left, north upward, and so on.

to strongly suggest local generation. Internal waves have elliptical particle orbits, with the major axis aligned with the horizontal wavenumber, and may be generated by similarly elliptical barotropic tidal orbits. All of the baroclinic inclinations are northwest, in the vicinity of $125^{\circ}$, indicating horizontal wavenumber in the direction $325^{\circ} \mathrm{T}$, approximately along the mooring line. This is in rough agreement with the barotropic inclinations, particularly for diurnal constituents at S5a and S4. The baroclinic parameters for the S5a and S4 sites are similar to each other and are all consistent with waves propagating from S5a to S4, but they are very different from those of S7. The discontinuity is strongest for the diurnal constituents, which are highly similar at S4 and S5a but differ in amplitude and direction at S7. This discontinuity, also evident in Fig. 12, suggests diurnal internal tide generation between S7 and S5, which we will discuss in the next section. One caveat to mention is that diurnal oscillations are evident deep at S7, so that diurnal waves may be coming into the area from the south, but those few phase and inclination estimates are not stable over depth and do not make enough sense when compared with S5a and S4 parameters to determine whether that is happening.

The S5a and S4 baroclinic phases in the table are consistent with a progressive wave moving northwest in the direction of the mooring line, breaking the northwest/southwest ambiguity of the inclination and allowing estimation of horizontal wavenumber vectors. The diurnal phases change from 201 to $230^{\circ}(\mathrm{O} 1)$ and from 310 to $336^{\circ}$ (K1) between S5a and $\mathrm{S} 4$, suggesting that the moorings are 0.080 and 0.072 horizontal wavelengths apart for the two constituents. This yields wavelengths $2 \pi / k_{h}$ of 106 and $117 \mathrm{~km}$, respectively. The corresponding phase velocities are $1.14 \mathrm{~m} / \mathrm{s}$ for $\mathrm{O} 1$ and $1.35 \mathrm{~m} / \mathrm{s}$ for $\mathrm{K} 1$. These are comparable to the mode-one $\mathrm{O} 1$ phase speed estimates given earlier (Fig. 10 caption). For semidiurnal M2, the phase changes from $67^{\circ}$ to $165^{\circ}$, or 0.272 wavelengths, between S5a and S4, yielding a wavelength of $31 \mathrm{~km}$ and a phase speed of $0.70 \mathrm{~m} / \mathrm{s}$. 
TABLE II

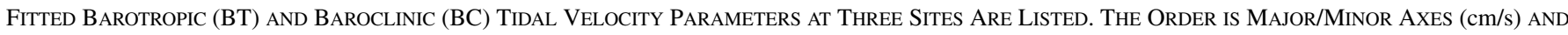
InClinATION/PhASE (DEGREES). BC PARAMETERS FOR NEAR-SuRFACE CURRENTS ARE UsED. CONFIDENCE INTERVALS FOR O1 AND BT S2 QuANTITIES ARE 30

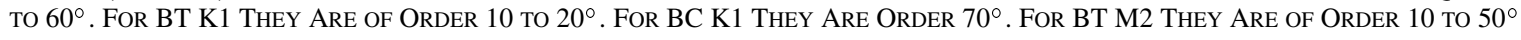

\begin{tabular}{c|ccc}
\hline Constituent & S7 & S5a & S4 \\
\hline BT Diurnal O1 & $6.3 /-0.7173 / 56$ & $9.3 /-3.0125 / 27$ & $8.9 /-4.9121 / 19$ \\
BT Diurnal K1 & $4.8 / 1.1162 / 84$ & $10.1 /-3.7137 / 66$ & $11.9 /-5.8137 / 59$ \\
BT Semidiurnal M2 & $3.6 /-2.1154 / 351$ & $5.5 /-4.982 / 55$ & $9.0 /-5.1116 / 38$ \\
BT Semidiurnal S2 & $1.2 /-0.9166 / 2$ & $2.5 /-1.0109 / 86$ & $3.6 /-2.786 / 99$ \\
\hline BC Diurnal O1 & $8.4 /-3.5104 / 165$ & $16.2 /-11.8132 / 201$ & $14.2 /-10.8122 / 230$ \\
BC Diurnal K1 & - & $11.2 /-9.0133 / 311$ & $11.1 /-8.9120 / 336$ \\
BC Semidiurnal M2 & $9.5 /-3.0137 / 308$ & $7.6 /-3.3129 / 67$ & $5.1 /-2.092 / 165$ \\
BC Semidiurnal S2 & $6.0 /-3.6133 / 313$ & - & $3.9 /-3.0117 / 110$ \\
\hline
\end{tabular}

To summarize this section, tidal fitting to isotherm displacements reveals strong internal tides. Fitting to baroclinic velocity time series provides estimates of internal tidal amplitudes, inclinations and phases. These diurnal parameters vary over depth in a manner consistent with upward propagating internal waves (downward phase velocity), moving to the northwest, being present at all sites. The diurnal signals have larger amplitudes at the shallower sites, and at S7 they are larger near the bottom than near the surface. The inclinations and phases are consistent with diurnal waves of 100-km scale and semidiurnal waves of $30-\mathrm{km}$ scale moving to the northwest from S5a at quantifiable phase velocities. The semidiurnal parameters indicate weaker waves that do not have the same clear downward phase velocity, and may have upward phase velocity. The internal tide phase velocities are lower than the speeds of high-frequency nonlinear internal waves presented in Section IV.

\section{INTERNAL TIDE DISCUSSION}

The most notable internal tidal waves that we have measured are the diurnal waves at the shallower S5a and S4 sites, which stand out in the time series. The spring-neap cycling causes two constituents to be fit, $\mathrm{O} 1$ and $\mathrm{K} 1$, but the partition of energy between them is unreliably computed. Because internal waves are subject to advection and Doppler-shifting, and because generation is sensitive to stratification that may change over time, it is possible that the relative amplitudes and phases of the two components may not be stable over mesoscale time periods. However, it is likely, although not certain, that the spring-neap cycle of dominantly diurnal internal tides is a stable feature of the area.

Although the fitting procedure does not give highly reliable estimates of internal tide parameters, it does allow us to separate the internal tidal signals from other internal wave signals and from the barotropic tidal signals. The resulting approximations to the actual internal tidal waves can be analyzed, letting us characterize the waves.

We will address five questions about the diurnal internal tides in this section: (1) How do the wave motions compare with linear internal wave solutions? (2) Where are the waves generated? (3) What becomes of the waves in shallow water? (4) What is their energy flux? (5) Are they nonlinear and does this effect their propagation?

\section{A. Phase Relationships}

Phase can be analyzed to evaluate whether or not the fitted baroclinic currents and displacements are consistent with linear internal waves. Phase relationships for internal wave velocity components are evident in the expressions for velocity $u_{r}$ in the wavenumber direction, the component $v_{r} 90$ degrees anticlockwise from there, the vertical velocity $w$ and the displacement $\eta$, which are

$$
\begin{aligned}
w & =\frac{-\kappa \omega}{\left(N^{2}-f^{2}\right) \sin \phi} \frac{p^{\prime}}{\rho_{0}} \\
u_{r} & =\frac{\kappa \omega}{\left(N^{2}-f^{2}\right) \cos \phi} \frac{p^{\prime}}{\rho_{0}} \\
v_{r} & =\frac{-i f u_{r}}{\omega} \\
\eta & =\frac{-i w}{\omega}
\end{aligned}
$$

where $\kappa$ is the magnitude of the wavenumber vector, $\omega$ is the wave frequency, $\phi$ is the angle that the wavenumber vector makes with the horizontal, $\rho_{0}$ is background density, and perturbation pressure $p^{\prime}$ has the form $\exp [i(k x+l y+m z-\omega t)]$ [26]. The first three equations apply to a point in Eulerian coordinates, but the fourth does not.

The sine of the angle $\phi$ is negative for a wave with downward wavenumber $\mathbf{k}=(k, l, m)$ (negative $m$ ) and downward phase velocity, which has an upward group velocity. It is positive for upward $\mathbf{k}$ (positive $m$ ). This means that $w$ and $u_{r}$ have the same sign for downward $\mathbf{k}$, and opposite sign for upward $\mathbf{k}$. An important result is that $\eta$ and $v_{r}$ are in phase for downward $\mathbf{k}$ and $180^{\circ}$ out of phase for upward $\mathbf{k}$. The lag between $u_{r}$ and $v_{r}$ is always $90^{\circ}$.

Because of phase convention, the advancing phase with depth seen in the diurnal current constituents is consistent with downward $\mathbf{k}$, so $\eta$ should follow $u_{r}$ by $90^{\circ}$. This is not precisely true of the parameters obtained for individual constituents, even for the strongest signals that dominate the time series (to the eye), which are the diurnal signals at S5 and S4. Fig. 20 shows displacement and current time series at approximately 50-m depth at the 200-m depth site. The phases at S5a for $\eta_{23}$ are $341^{\circ}$ for $\mathrm{O} 1$ and $137^{\circ}$ for $\mathrm{K} 1$. These are advanced 128 and $161^{\circ}$ from the $u_{r}$ phases of the fitted $\mathrm{O} 1$ and $\mathrm{K} 1$ currents at the mean depth of this isotherm, $53 \mathrm{~m}$. The sign of the phase advance is correct, 


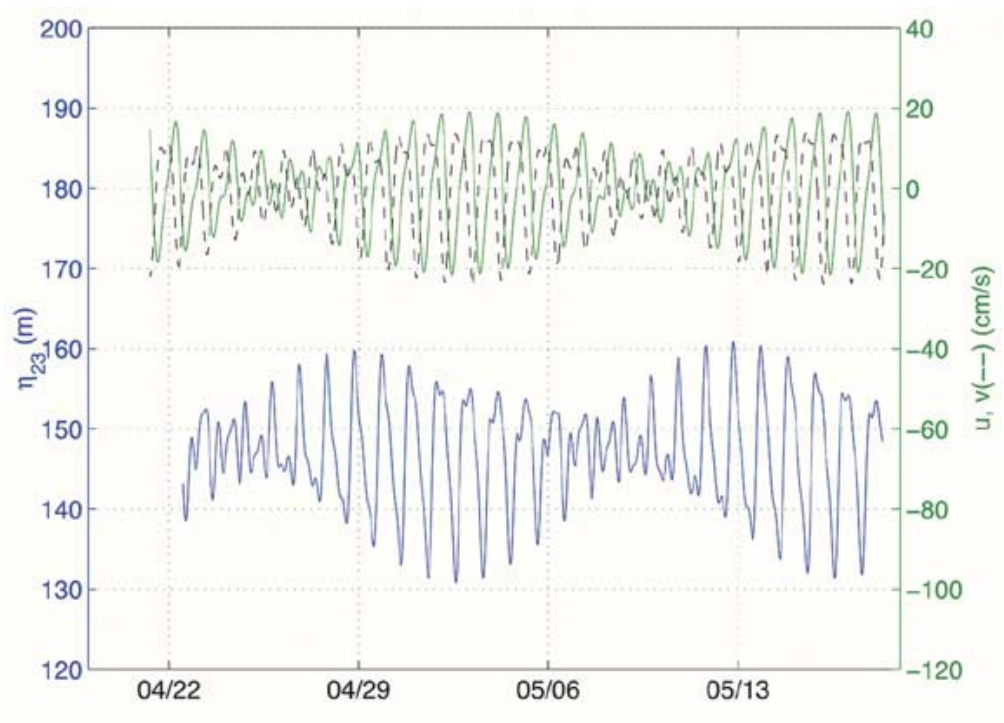

Fig. 20. Fitted S5a tidal series for $u, v$ at 53-m depth and for $\eta_{23}$ measured at S5 are shown. The velocities are at the top ( $v$ dashed line) with units indicated on the right. Height above the bottom of the $23^{\circ}$ isotherm $\left(\eta_{23}\right)$ bottom is shown below with units on the left.

but the amount is exaggerated. The $\eta$ and current time series, consistent with nonlinearity of the internal tide, is not well fit with tidal constituents, although some of the skewness can be captured by the so-called shallow-water constituents. Examples of these are the M3 and M4 overtones of M2. The steppy nature or skewness is also not consistent with (1) through (4), so it is no surprise that the phases are not correct. The phase errors may be a useful measure of internal tide nonlinearity.

The ability to fit tidal constituents to large fractions of the tidal-band displacement and baroclinic current variances $(50 \%$ for the currents and $55 \%$ for $\eta_{23}$ shown in Fig. 20) is remarkable. Although not shown, the time series of $w^{\prime}$ computed by differentiating the tidal fit to $\eta_{23}$ corresponds very well with $u^{\prime}$ during many intervals.

\section{B. Possible Diurnal Generation Site}

The baroclinic diurnal tidal parameters at S4 and S5a show larger currents than at S7, have consistent inclination to the northwest, show lateral phase progression consistent with waves of the expected wavelength, and have vertical phase relationships that are consistent with propagation upward along sloping characteristics. The S7 inconsistency suggests that the waves are generated between S5 and S7. This cannot be conclusively shown with the data analysis performed to date, but we show here that the area is suitable for diurnal tidal generation.

Bottom slopes $(\alpha)$ equal to the slope of characteristics, called critical slopes, have been linked to internal wave generation. In the analytic analysis of tide generation at slopes by Baines [27], internal tide amplitudes generated over a linear slope are expected to be small unless $(1-\alpha / C) /(1+\alpha / C)$ is small, for the case of approximately constant $C$ (or $N$ ). This mean that $\alpha / C$ must be near unity, the near-critical situation. Table III gives critical slope values for semidiurnal and diurnal internal waves at three values of $N$ typical of this region. The lower value of $N$ is expected near the bottom. The semidiurnal critical slopes are of order 0.5 to 1 degree, the diurnal critical slopes are 0.16 to 0.3 degrees.
TABLE III

INTERNAL WAVE RAY CHARACTERISTIC ANGLES, IN DEGREES FROM THE HORIZONTAL, AT TWO WAVE FREQUENCIES

\begin{tabular}{c|ccc}
\hline Constituent & $N=4 \mathrm{cph}$ & $N=8 \mathrm{cph}$ & $N=12 \mathrm{cph}$ \\
\hline Diurnal O1 & 0.32 & 0.16 & 0.10 \\
Semidiurnal M2 & 1.0 & 0.53 & 0.35 \\
\hline
\end{tabular}

Only the shallower slopes that are critical for diurnal waves are typical of this region; those critical for semidiurnal are not. To illustrate this, Fig. 21 shows slopes mapped from bathymetry data collected by a number of vessels during ASIAEX. The data obtained along ship tracks were interpolated onto a grid of order kilometer spacing, then smoothed with a 3-by-3 point 2-D Wiener filter. The filtering was intended to reject noise from ship motion, tides, and calibration differences between vessels. The gradient of the resultant field was computed, and then subjected to the same Wiener filter. The figure shows relatively flat areas with slopes less than 0.5 degree and small regions with steeper slope. The steep regions are depicted to be larger than they actually are because of the filtering; they are typically have lateral scale of $200 \mathrm{~m}$. There is little area with slope between 0.5 and $1.0^{\circ}$. The mapped slopes in the area between S5 and S7 agree well with estimates obtained along individual ship tracks, but some of the details in the figure are artifacts. Thus, much of the sea floor has a slope close to that of a typical diurnal characteristic, but a slope less than that of a typical semidiurnal characteristic (i.e., the sea floor is diurnal critical, semidiurnal subcritical).

To further explore the possibility of generation at this site, consider that internal wave orbital velocities are elliptical in a plane parallel to characteristics (normal to the wavenumber), with the major axis and the wavenumber aligned horizontally [see (1)-(3)]. Sinusoidal barotropic tidal currents forced to have a vertical component in order to match the no-normal-flow bottom boundary condition would have currents like this if the current ellipses have the correct major/minor axis ratio, the 


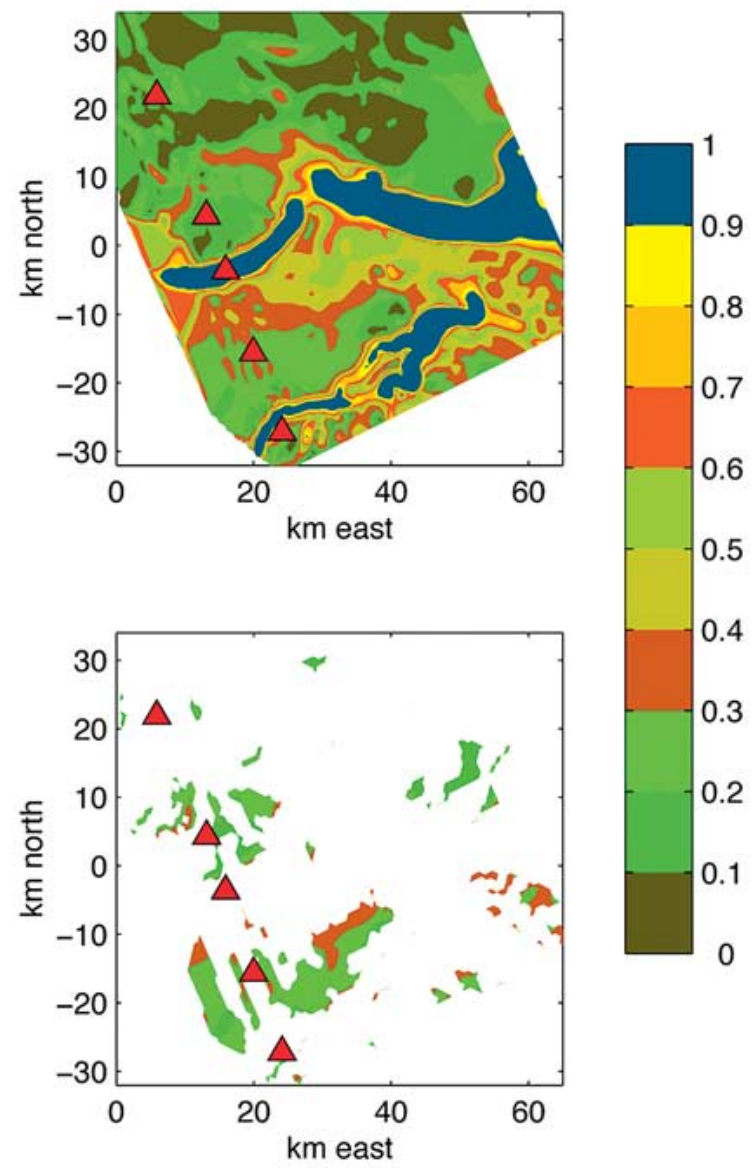

Fig. 21. (top) Contour diagram of bottom slopes of the area, in degrees, is shown. Spatial filtering has been applied to reduce noise at the expense of resolution. All values above 0.9 are blue. (bottom) Bottom slopes are again contoured, but only those regions with slopes between 0.15 and $0.4^{\circ}$ and with upslope direction between $300^{\circ}$ and $360^{\circ}$ (north) are included.

currents rotate anticycloniclly, and the major axis is pointed up a critical slope. Note that these vertical velocities attenuate with height above bottom, so the orbits may also match internal wave orbits high above supercritical slopes. Interfering barotropic waves producing a simple orbital flow over a few wave periods, as is evident in the lower panel of Fig. 13, may also produce transient particle orbits like those of linear internal waves, unlike the complicated erratic patterns sometimes produced by interfering mixed tidal waves. The S5a diurnal barotropic currents are anticyclonic, have suitable ellipticity, and the major axes have inclinations of 132 and $133^{\circ}$ (317 and $318^{\circ}$ true). Regions with near-critical slopes aligned in this direction are likely to radiate diurnal internal waves, with the waves traveling in the horizontal direction given by the barotropic inclination.

The lower panel of Fig. 21 shows areas where slopes are in the critical diurnal range and have direction between $300^{\circ}$ and $360^{\circ}$. The large direction window is necessary because the mapped slope field is noisy. There is a large area between 10 and $40-\mathrm{km}$ east and -30 and $-10 \mathrm{~km}$ north, between S5 and $\mathrm{S} 7$, meeting these conditions, which is judged to be suitable for generation of the observed internal tidal waves by the observed barotropic tidal waves. The gaps in this larger colored zone (near S6) are probably due to repeated ship tracks along the mooring lines giving inconsistent depths, thus resulting in slope artifacts. Baroclinic tidal analysis at S7 shows mid-depth diurnal motion consistent with internal waves having upward group velocity (Fig. 14), so it is also possible that the waves are generated south of our work area, as mentioned earlier. In addition to inconsistencies in the deep S7 tidal parameters, the bathymetry is not known well enough out of our immediate work area to determine slope, so generation to the south can't be investigated with existing data.

Besides slope criticality, another constraint on the effectiveness of the generation of internal tides, specifically an interfacial mode internal tidal wave at the edge of the continental shelf, is that the parameter $T$ be less than or equal to 2 , where $T=$ $h_{l} / L \alpha, h_{l}$ is the depth of the continental shelf and $2 \pi L$ is the horizontal wavelength [27]. Defining mixed-layer (upper layer) thickness to be $d$, and defining the gravitational restoring force at the interface with density jump $\delta \rho$ located at the base of the mixed layer to be $g^{\prime}=g \delta \rho / \rho_{0}$, we have $L^{2}=g^{\prime} d /\left(\omega^{2}-f^{2}\right)$. This can be evaluated for the stratification and bathymetry of this area at both diurnal and semidiurnal frequencies $\omega$. Using (hypothetical) near-critical slope in each case (angles 0.2 and $0.8^{\circ}, \alpha=0.0035$ and 0.0140$), d=40 \mathrm{~m}, h_{l}=110 \mathrm{~m}$ and $g^{\prime} / g=0.001$, we have $T$ values of 2.0 and 1.6 for the diurnal and semidiurnal cases. Note that the above numbers give $2 \pi L$ of 30 and $100 \mathrm{~km}$ for semidiurnal and diurnal waves, respectively, as observed, although the expressions are for interfacial waves.

The hypothesis that diurnal waves are ascending from near the bottom can be checked by looking at velocity data from site S6 at 275-m depth, which is in the center of the hypothesized generation zone. This current meter did not cover enough of the water column to allow computation of reliable barotropic tidal current, so tidal analysis could not be performed on baroclinic residuals, as was done with S7, S5a and S4 data. However, untreated S6 time series of northward ( upslope) velocity $v(t, z)$ (Fig. 22) clearly show diurnal oscillations in the lower half of the water column during the May 1-6 time period when large diurnal waves were seen at S5a and S4, agreeing with this hypothesis. Semidiurnal oscillations concentrated near the surface can be seen during this time interval. This figure showing currents without processing of any type illustrates the dominance of the baroclinic tidal signals and the complicated nature of the tidal band flow field. The field consists of distinct semidiurnal and diurnal baroclinic waves in addition to the many barotropic constituents and high-frequency waves.

\section{Wave Propagation}

The diurnal internal tides that are evident in the S5 and S4 data undergo a transition as they propagate. Fig. 23 shows the development of the shoaling diurnal wave measured on May 3, a day of active diurnal internal tide. The total currents in near-surface layers at S7, S5a and S4 are shown in the upper three panels, rotated $110^{\circ}$ anticlock-wise, so that $u_{r}$ in the assumed wave direction $340^{\circ}$ is shown in red, and $v_{r} 90^{\circ}$ to the left is shown in blue. The peak in $u_{r}$ can be followed from S7 to S5a to S4 and is indicated with the gray boxes. Clock-wise current rotation can be seen. The currents show that the wave steepens into a bore-like feature as it moves from S5a to S4. This can also be seen in S4 isotherms, contoured in the bottom panel. The bore 


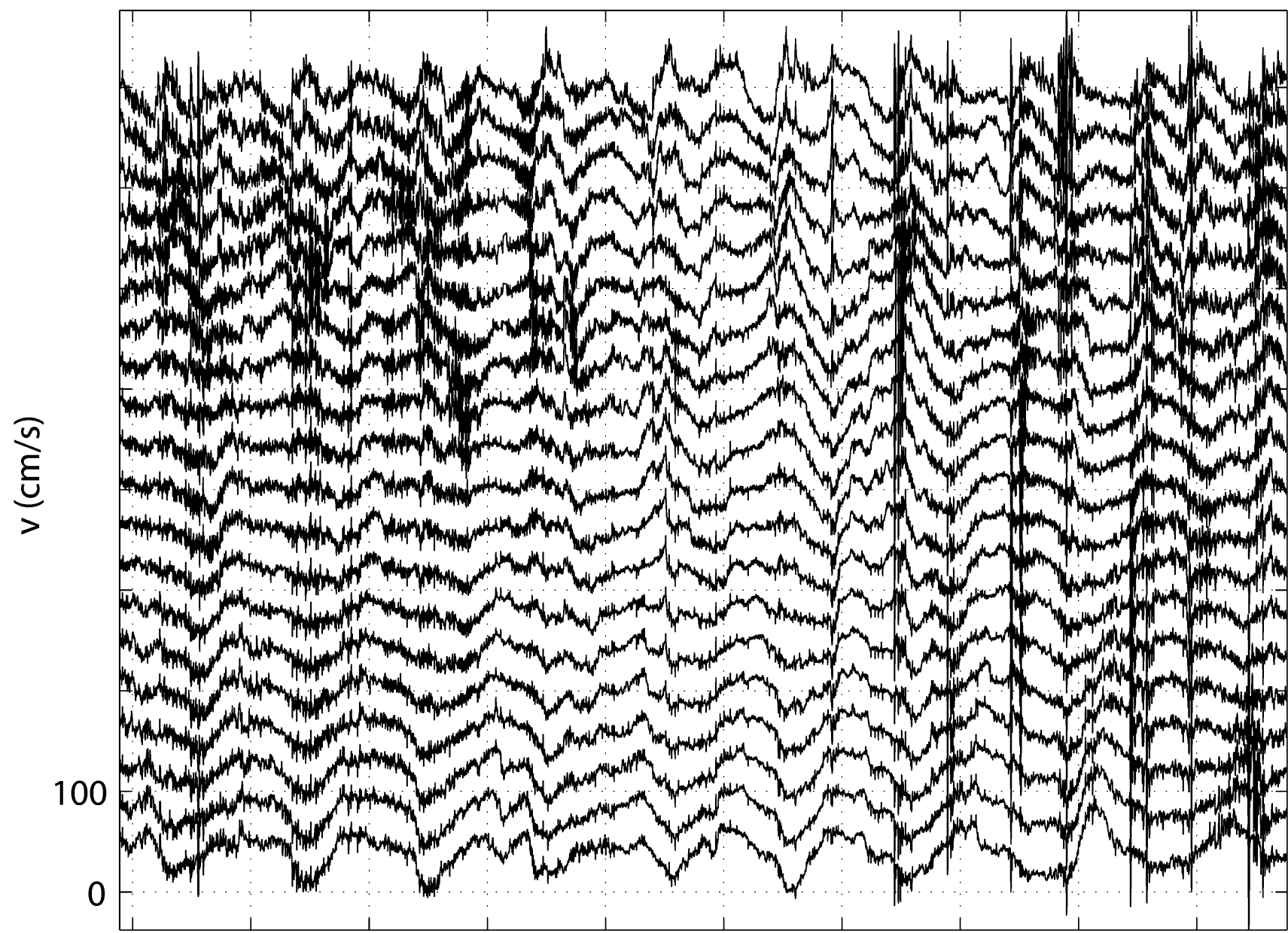

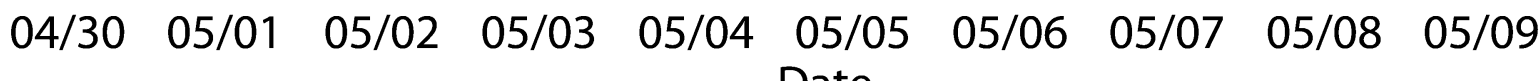
Date

Fig. 22. S6 northward velocity. The currents are from 30,40,50, . $220 \mathrm{~m}$ depth, and are offset so that they can all be seen. The 30-m velocity is at the top.

can be seen at $\mathrm{S} 4$ at time 3.5, with small 10- to 15 -m amplitude solitary-type internal waves accompanying the bore. The ADCP does not reliably detect the upper-layer currents of these small waves. These data are also shown in Fig. 4, where it can be seen that although the diurnal internal tidal wave steepens between $\mathrm{S} 5$ and S4, there is a half-day period internal wave present at S7 between 0000 and 1800 of May 3 that seems to be breeding solitary-type waves, seen around 0600. These are not well measured by the ADCP so their direction isn't easily known, although the S7 M2 semidiurnal internal tide has inclination $130^{\circ}$ (Fig. 17), which suggests wavenumber directed at $320^{\circ}$.

The current data and the temperature (density) data from S4 can be used to compute energy flux in the diurnal wave. The energy flux of summed barotropic and baroclinic tidal waves would be

$$
\mathbf{F}=\int d z\left\langle\left(\mathbf{U}^{\prime}+\mathbf{u}^{\prime}\right)\left(P^{\prime}+p^{\prime}\right)\right\rangle
$$

where $\mathbf{U}^{\prime}$ is barotropic wave velocity, $P^{\prime}$ is barotropic pressure anomaly due to surface displacement, $\mathbf{u}^{\prime}$ is baroclinic wave velocity in a fixed reference frame, and $p^{\prime}$ is baroclinic wave pressure anomaly. The brackets indicate average over one wave pe- riod. The flux breaks into four parts. One is consistent with a purely barotropic wave. Another is consistent with a purely baroclinic wave, with integrand $\left\langle\mathbf{u}^{\prime} p^{\prime}\right\rangle$. The other terms have factors from both waves. We will compute the energy flux for the baroclinic wave here, disregarding the cross terms, since we are more interested in characterizing the internal tide rather than in exhaustively studying the energy budget.

Computing $p^{\prime}(z, t)$ by integrating density from the surface at each time $t$, subtracting $\mathbf{U}^{\prime}$ from the total current to obtain an estimate of $\mathbf{u}^{\prime}$, and integrating $\left\langle\mathbf{u}^{\prime} p^{\prime}\right\rangle$ over depth and over one O1 period starting at 0000 on May 3, we obtain energy flux of $1300 \mathrm{~W} / \mathrm{m}$ in the direction $47^{\circ}$ for the data shown in Fig. 23. For this calculation, a rough estimate of the surface displacement $\eta_{s}$ of the internal wave is included. The estimate is obtained based on two-layer baroclinic wave expressions [26], adjusted to account for continuous stratification, despite the fact that the waves are observed to not be modal. The expression we use is $\eta_{s} / \eta_{24}=\left(-g^{\prime}\right) / g \pi$, where $g^{\prime}=g\left(\rho_{2}-\rho_{1}\right) / \rho_{2}$ is the reduced gravity between the two layers of densities $\rho_{1}$ and $\rho_{2}$. The equivalent expression for the two-layer case does not contain the factor of $\pi^{-1}$, which is included to approximate the solution for the case of constant density gradient, derived 


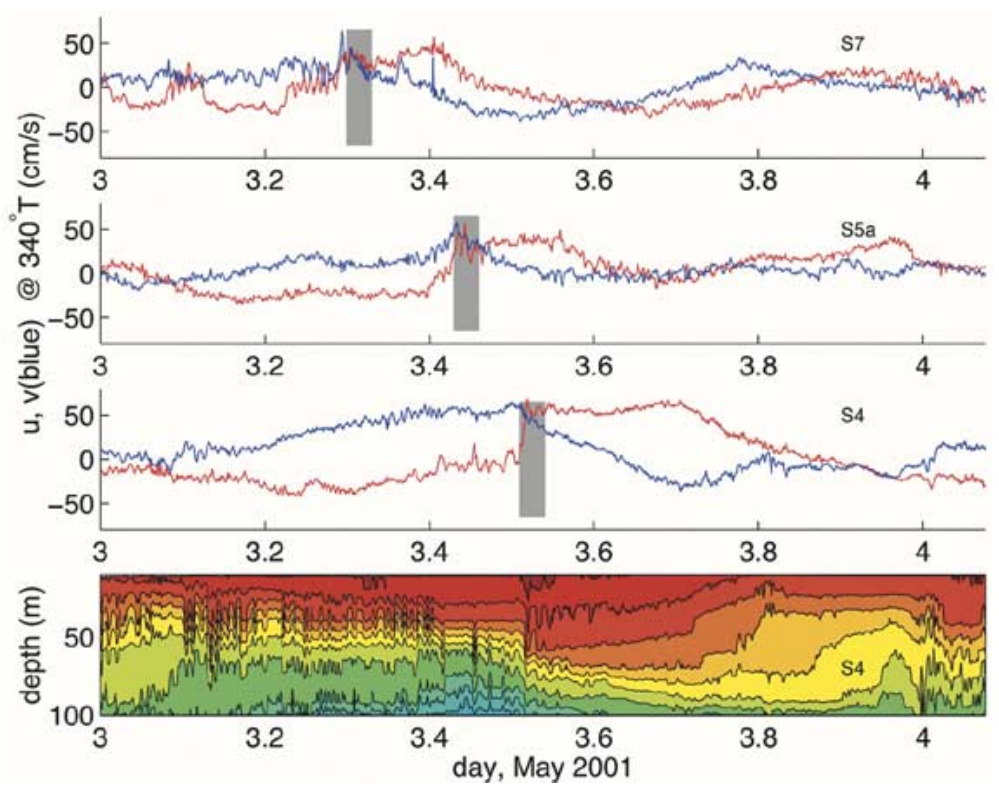

Fig. 23. Upper layer currents, rotated to the approximate internal tide heading of $340^{\circ}$ true, are plotted for three stations: S7 (top), S5a (2nd from top), and S4 (3rd from top). S4 Isotherms contoured with one degree interval are also shown (bottom). One O1 tidal period is included (25.82 h). Currents $u_{r}$ (red) and $v_{r}$ (blue) point in the direction of the horizontal wavenumber and $90^{\circ}$ anticlock-wise from the wavenumber, respectively. The gray boxes indicate intervals of peak current in the propagation direction of $340^{\circ}$ moving from S7 to S4. The approximately sinusoidal $u_{r}$ seen at S7 evolves to have a more peaked form at time 3.43 at S5a. At S4 it has further evolved into a bore-like form. The S4 isotherms show the downward progression of phase seen in previous figures (wave arrives later as depth increases), and also shows a number of 20-m amplitude nonlinear internal waves traveling with the bore that are not detected by the ADCP.

by J. Colosi for finite length waves (J. Colosi, personal communication). The pressure anomaly from the displacement is $g \rho_{1} \eta_{s}$, with $\rho_{1}=1021.5 \mathrm{~kg} / \mathrm{m}^{3}$, with typical displacements of a few centimeters. Including these estimates of $\eta_{s}$ reduces bottom pressure anomalies to a very low level, consistent with our bottom pressure observations showing close agreement with tidal surface height predictions.

This computation was repeated each day, giving small or unreliable flux estimates on the days when diurnal internal tides were not dominant (see Fig. 12), and a maximum northward flux of $1650 \mathrm{~W} / \mathrm{m}$ at $328^{\circ}$ on May 16. (A $u^{\prime}, p^{\prime}$ cross-spectral method of estimating flux within tidal bands may offer an improvement over this approach.) Computed flux is to the south for a few days of dominant diurnal tides, but the vector mean diurnal flux over the entire deployment is $1000 \mathrm{~W} / \mathrm{m}$ at $330^{\circ}$. (An alternative calculation using $p^{\prime}$ computed using density anomaly only, i.e., no surface displacement, always gave northward flux.) The abrupt changes in computed flux suggest that the surface displacement estimate is inadequate. The cycle over the duration of the experiment (not shown) is as expected from inspection of Fig. 12. Semidiurnal and high-frequency waves dominate estimates for April 23-28, May 6-8, and May 14. Good diurnal estimates showing northward flux were obtained for April 29, May 3-5, May 12-13, and May 15-16. Dominantly diurnal days April 30-May 2 have uncertain flux directions that are sensitive to the details of $\eta_{s}$ estimation. The behavior of $u^{\prime} p^{\prime}$ over time and space has a reproducible signature. The double integral is often dominated by the product of negative $u^{\prime}$ and negative $p^{\prime}$ (velocities opposed to the direction the wave is traveling, and downward displaced isopycnals) occurring underneath strong positive $u^{\prime}$ in the upper layer, which occurs just after the passage of the bore (the rapid downward displacement of the thermocline).

The maximum diurnal internal tidal flux is about six times higher than the maximum M2 internal tidal energy fluxes computed using models of the North West Australian Shelf [29]. The maximum flux of the diurnal internal tide that we have estimated is close to the transbasin wave energy flux inferable from measurements of Orr and Mignerey. They estimated $90 \mathrm{MJ} / \mathrm{m}$ energy per unit along-crest length in a $5-\mathrm{km}$ zone (normal to the crests) of an energetic packet. Dividing this number by the half-day interval between packets gives $2080 \mathrm{~W} / \mathrm{m}$ average flux rate.

Because the wave is moving directly along the mooring line its speed can be measured. The time it takes the $u_{r}$ peak to travel between S5a and S4 gives speed $1.12 \mathrm{~m} / \mathrm{s}$. This gives a horizontal wavelength of $104 \mathrm{~km}$ for an internal wave of O1 frequency. If $N=6$ is assumed, a vertical wavelength of $390 \mathrm{~m}$ can be deduced, which is very close to double the depth at the deeper site. This horizontal wavelength and this phase speed are in good agreement with the tidal analysis estimates that were given in Section VI.

\section{Nonlinearity}

The conversion of the internal waves to bores and packets of short waves, such as we have shown on May 3 at the S4 site, shows that the waves are nonlinear. The increased energy density expected as the internal waves travel into shallow water is a possible cause of the large velocities and nonlinearity.

The situation may be compounded at the surface for the diurnal waves. The apparent downward wavenumber for the diurnal wave means that group velocity is upward, and that 


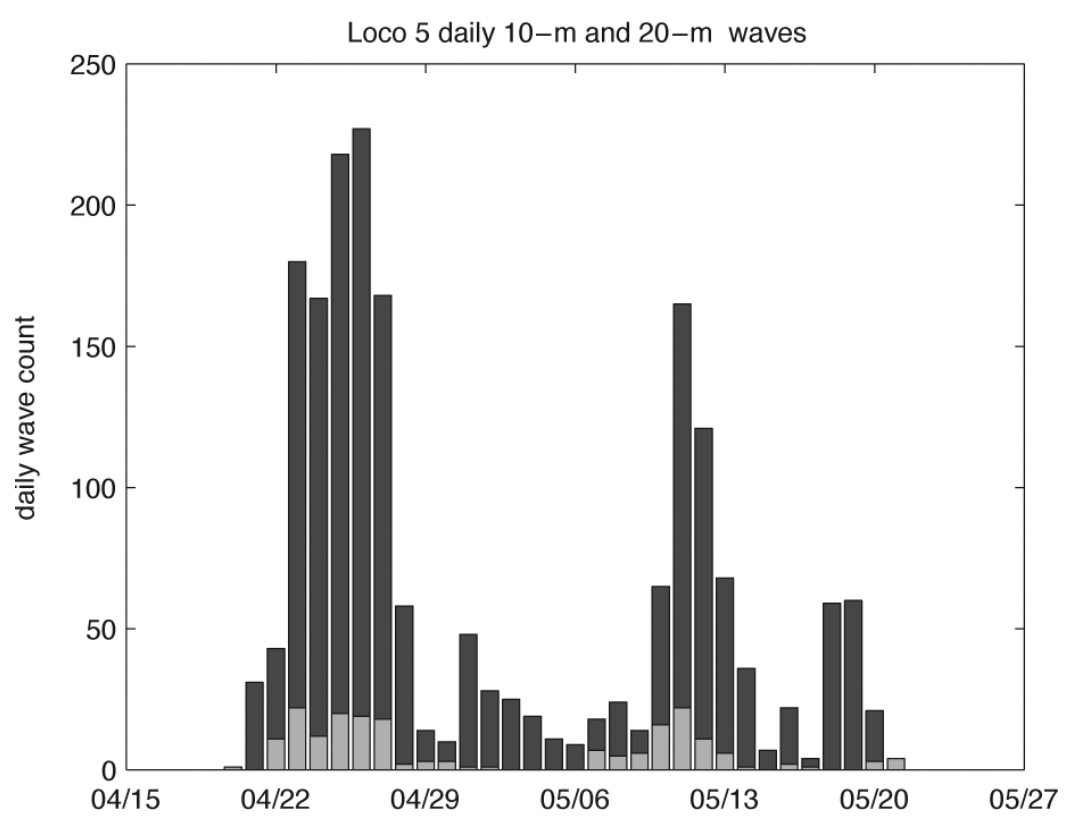

Fig. 24. Daily quantities of $10 \mathrm{~m}$ or greater amplitude internal waves of $10 \mathrm{~min}$ or less duration at Loco 5 are shown with the black bars. The daily counts of $20 \mathrm{~m}$ or greater amplitude waves of $10 \mathrm{~min}$ or less duration are shown with gray bars.

particles move upward as they move forward in their orbits. This is not possible for waves in the process of reflecting at the surface (or from the bottom of the mixed layer), however, and the upper layers may have phase inconsistencies and deformation associated with the reflection. A difference between behavior at the surface and below is evident in Fig. 11, where the isotherms trace smooth undulations near the bottom but have sharp discontinuities near the surface.

The ratio of particle velocity to phase velocity $u_{r} / c$ is one measure of nonlinearity. The orderly structure of the shallow area wavefield when it was dominated by the diurnal wave offers some hope of computing this. Consistent phase velocities have already been determined with two methods, tidal analysis and the observation of patterns at S5a and S4. Tidal analysis also served to isolate tidal internal wave particle velocities from other nontidal signals. Fig. 13 shows a selection of these. The $u_{r}$-components of the largely diurnal fitted S5a velocities, rotated into the observed wave direction of $340^{\circ}$, can be divided by the estimated phase speed of $1.12 \mathrm{~m} / \mathrm{s}$ to obtain time series of $u_{r} / c$. These have peak values of 0.16 and -0.24 during the period surrounding May 3, the day of the illustrated steepening wave. These values are much less than unity, but are nonetheless sufficiently large to be associated with nonlinear effects.

Features very similar to the bore accompanied by short internal waves, seen in the lower panel of Fig. 23, have been observed on the Mid-Atlantic Bight shelf of the western Atlantic Ocean [18], [19], and at other locations. They appear with only semidiurnal periodicity at the Atlantic site because the diurnal frequencies are below the internal wave band lower limit $f$ at the more northerly latitude of that site. It is very likely that the Atlantic bores and waves evolved in the manner demonstrated clearly here with this large data set.

The steepening of the internal tide means that there are two sources of high-frequency nonlinear waves on the shelf in the northern area of ASIAEX operations, the transbasin waves and the internal tide. Fig. 24 shows the daily quantity of short-period (10-min) internal waves measured directly by isotherm motion past sensors on Loco 5. No interpolations or assumptions are required to detect waves exceeding $10-\mathrm{m}$ amplitude with sensors $10 \mathrm{~m}$ apart, and the same is true for $20-\mathrm{m}$ waves and spacing. The figure shows that waves exceeding 20-m amplitude can be associated with the two periods of transbasin waves, with a gap between the two periods. On the other hand, waves of $10-\mathrm{m}$ amplitude occur on all days, and result from at least these two processes.

\section{RESULTS}

Internal gravity wave data, obtained during a 1-mo long field program with moorings located at the edge of the continental shelf in the northern SCS, have been presented. Two processes evident in the data have been examined in detail: Passage on to the shelf of wave energy originating in high-amplitude high-frequency nonlinear internal waves of depression; and long-wavelength internal tidal wave propagation.

The high-amplitude (transbasin) waves of depression were observed to change from waves of quasi-permanent form in water deeper than $350 \mathrm{~m}$ to waves of depression of much longer horizontal scale in water of 120 to $80 \mathrm{~m}$ depth. The front faces of the waves moved as bores in shallow water, apparently steepening into bores after passing through a stage having a shallow front face and steep rear face (at least in the case of the largest waves). Trailing behind the ever-lengthening depression waves in shallow water were tails of short-scale waves apparently fed by energy from the incident wave. Although we have not yet done detailed comparisons between observed wave shapes and theoretical wave shapes, the waves appear to be somewhat consistent with weakly nonlinear theory, until dissipation processes become dominant. A reason for this consistency, despite the large wave amplitudes with respect to the upper layer depth, may 
be that instability processes prevented the waves from reaching high enough energy density and high enough steepness to prevent the use of weakly nonlinear (KdV) theory, so long as the weak dissipation is properly parameterized [7], [20].

Prominent diurnal internal tides with a fortnightly cycle were measured in the shallow area of the experiment. The waves were consistent with waves moving to the northwest and upward in the water column. In deeper water, semidiurnal tides that appear to be moving down in the water column were evident near the surface, with diurnal oscillations seen closer to the bottom. The relationships between parameters estimated at different stations suggest local generation of the diurnal internal tide in the midst of the mooring array, with the semidiurnal tide moving into the area from the southeast, but the local diurnal generation and the semidiurnal tide radiation can not be confirmed without observations offshore of those reported here. A possible diurnal internal tide generation site having critical slope was identified within our measurement area. Finally, nonlinearity of the diurnal internal tide and conversion into a bore with small solitary waves was demonstrated.

Quantitative values were obtained for a number of diurnal internal tide features. Horizontal wavelength $2 \pi / k_{h}$ was of order $110 \mathrm{~km}$, horizontal phase velocity was $1.2 \mathrm{~m} / \mathrm{s}$, vertical wavelength $2 \pi C / k_{h}$ was of order $400 \mathrm{~m}$, or twice to four times the depth. Energy flux peaked at about $2000 \mathrm{~W} / \mathrm{m}$ during our $1 \mathrm{mo}$ of observations, and averaged about half that.

A study of baroclinic tidal parameters and of velocity coherences along internal wave characteristics would improve upon this paper. The internal tide generation and propagation problem is a 3-D puzzle, and further analyses of these data may uncover more details of the observed baroclinic tidal waves.

\section{ACKNOWLEDGMENT}

Many mooring and instrumentation professionals contributed to this extensive field effort. J. Kemp, N. McPhee, L. Costello, W. Ostrom, J. Dunn, and A. Newhall from Woods Hole Oceanographic Institution (WHOI); M. Stone and F. Bahr from the Naval Postgraduate School, Monterey, CA (NPS); and W.-H. Her and Y. Liu from National Taiwan University, Taipei, Taiwan (NTU). Most of the moorings were deployed by, and many were recovered with, the vessel Ocean Researcher 1 of NTU. The highly competent work of the captain and crew is greatly appreciated. The other mooring work was performed by the vessel Fisheries Researcher 1, whose captain and crew are also thanked. C. Jackson and F. Henyey offered helpful comments concerning solitary wave shapes. This is WHOI contribution number 10970.

\section{REFERENCES}

[1] C.-S. Chiu, S. R. Ramp, C. W. Miller, J. F. Lynch, T. F. Duda, and T.-Y. Tang, "Acoustic intensity fluctuations induced by South China Sea internal tides and solitons," IEEE J. Oceanic Eng., vol. 29, pp. 1249-1263, Oct. 2004.

[2] T. F. Duda, J. F. Lynch, A. E. Newhall, L. Wu, and C.-S. Chiu, "Fluctuation of $400 \mathrm{~Hz}$ sound intensity in the 2001 ASIAEX South China Sea expermient," IEEE J. Oceanic Eng., vol. 29, pp. 1264-1279, Oct. 2004.

[3] M.-K. Hsu and A. K. Liu, "Nonlinear internal waves in the South China Sea," Canad. J. Remote Sens., vol. 26, pp. 72-81, 2000.
[4] J. R. Apel, J. R. Holbrook, J. Tsai, and A. K. Liu, "The Sulu Sea internal soliton experiment," J. Phys. Oceanogr., vol. 15, pp. 1625-1651, 1985.

[5] S. R. Ramp, T.-Y. Tang, T. F. Duda, J. F. Lynch, A. K. Liu, C.-S. Chiu, F. Bahr, H.-R. Kim, and Y. J. Yang, "Internal solitons in the northeastern South China Sea part I: sources and deep water propagation," IEEE J. Oceanic Eng., vol. 29, pp. 1157-1181, Oct. 2004.

[6] M. H. Orr and P. C. Mignerey, "Nonlinear internal waves in the South China Sea: observation of the conversion of depression internal waves to elevation internal waves," J. Geophys. Res., vol. 108, p. 3064, 2003

[7] A. K. Liu, Y. Zhao, T. Y. Tang, and S. R. Ramp, "Model-data assimilation of internal waves in ASIAEX-2001," IEEE J. Oceanic Eng., vol. 29, pp. 1144- 1156, Oct. 2004

[8] V. D. Djordjevic and L. G. Redekopp, "The fission and disintegration of internal solitary waves moving over two-dimensional topography," $J$. Phys. Oceanogr., vol. 8, pp. 1016-1024, 1978.

[9] Q. Zheng, V. Klemas, X.-H. Yan, and J. Pan, "Nonlinear evolution of ocean internal solitons propagating along an inhomogenous thermocline," J. Geophys. Res., vol. 106, pp. 14 083-14094, 2001.

[10] J. Small, "A nonlinear model of the shoaling and refraction of interfacia solitary waves in the ocean. Part II: oblique refraction across a continental slope and propagation over a seamount," J. Phys. Oceanogr., vol. 31, pp. 3184-3199, 2001.

[11] V. Vlasenko and K. Hutter, "Numerical experiments on the breaking of solitary internal waves over a slope-shelf topography," J. Phys. Oceanogr., vol. 32, pp. 1779-1793, 2002.

[12] R. C. Beardsley, T. F. Duda, J. F. Lynch, S. R. Ramp, J. D. Irish, C.-S. Chiu, T. Y. Tang, and Y. J. Yang, "The barotropic tide in the northeast South China Sea," IEEE J. Oceanic Eng., vol. 29, pp. 1075-1086, Oct. 2004.

[13] G. Fang, Y.-K. Kwok, K. Yu, and Y. Zhu, "Numerical simulation of principal tidal constituents in the South China Sea, Gulf of Tonkin and Gulf of Thailand," Continental Shelf Res., vol. 19, pp. 854-869, 1999.

[14] R. Pawlowicz, R. Beardsley, and S. Lentz, "Classical tidal harmonic analysis including error estimates in MATLAB using T_TIDE," Computers Geosci., vol. 28, pp. 929-937, 2002.

[15] P. E. Holloway, E. Pelinovsky, T. Talipova, and P. Barnes, "A nonlinear model of internal tide transformation on the Australian North West Shelf," J. Phys. Oceanogr., vol. 27, pp. 871-896, 1997.

[16] G. B. Witham, Linear and Nonlinear Waves. New York: Wiley, 1974.

[17] A. K. Liu, "Analysis of nonlinear internal waves in the New York Bight," J. Geophys. Res., vol. 93, pp. 12317-12 329, 1988.

[18] J. A. Colosi, R. C. Beardsley, G. Gawarkiewicz, J. F. Lynch, C.-S. Chiu, and A. Scotti, "Observations of nonlinear internal waves on the outer New England continental shelf during the summer Shelfbreak PRIMER study," J. Geophys. Res., vol. 106, pp. 9587-9601, 2001.

[19] J. R. Apel, M. Badiey, C.-S. Chiu, S. Finette, R. Headrick, J. Kemp, J. F. Lynch, A. Newhall, M. H. Orr, B. H. Pasewark, D. Tielbuerger, A. Turgut, K. von der Heydt, and S. Wolf, "An overview of the 1995 SWARM shallow water internal wave acoustic scattering experiment," IEEE J. Oceanic Eng., vol. 22, pp. 465-500, 1997.

[20] A. K. Liu, Y. S. Chang, M.-K. Hsu, and N. K. Liang, "Evolution of nonlinear internal waves in the East and South China Seas," J. Geophys. Res., vol. 103, pp. 7995-8008, 1998.

[21] L. A. Ostrovsky and Y. A. Stepanyants, "Do internal solitons exist in the ocean?," Rev. Geophys., vol. 27, pp. 293-310, 1989.

[22] P. E. Holloway, E. Pelinovsky, and T. Talipova, "A generalized Korteweg-de Vries model of internal tide transformation in the coastal zone," J. Geophys. Res., vol. 104, pp. 18 333-18 350, 1999.

[23] D. Bogucki and C. Garrett, "A simple model for the shear-induced decay of an internal solitary wave," J. Phys. Oceanogr., vol. 23, pp. 1767-1776, 1993.

[24] K. R. Helfrich, "Internal solitary wave breaking and run up on a uniform slope," J. Fluid Mech., vol. 243, pp. 133-154, 1992.

[25] F. S. Henyey and A. Hoering, "Energetics of borelike internal waves," $J$. Geophys. Res., vol. 102, pp. 3323-3330, 1997.

[26] A. E. Gill, Atmosphere-Ocean Dynamics. Orlando, FL: Academic, 1982.

[27] P. G. Baines, "On internal tide generation models," Deep-Sea Res., vol. 29, pp. 307-338, 1982.

[28] S. J. Prinsenberg and M. Rattray, "Effects of continental slope and variable Brunt-Vaisala frequency on the coastal generation of internal tides," Deep-Sea Res., vol. 22, pp. 251-263, 1975.

[29] P. E. Holloway, "A numerical model of internal tides with application to the Australian North West Shelf," J. Phys. Oceanogr., vol. 26, pp. 21-37, 1996. 


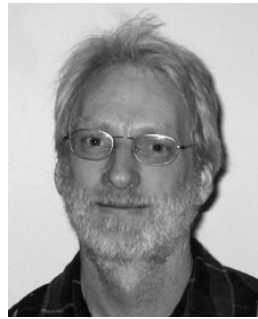

Timothy F. Duda received the B.A. degree in physics from Pomona College, Claremont, CA, in 1979 and the Ph.D. degree in oceanography from the Scripps Institution of Oceanography, University of California, San Diego, in 1986.

He has been a Scientist with the Woods Hole Oceanographic Institution (WHOI), Woods Hole, MA, since 1991. Prior to that, he held positions with the University of California, Santa Cruz. His three primary fields of study are ocean acoustic propagation, ocean internal gravity waves, and ocean mixing processes. He has modeled and analyzed fluctuations of ocean acoustic transmissions, has made electromagnetic-type internal-wave measurements, and performed in situ measurements of ocean microstructure.

Dr. Duda is a Member of the American Meteorological Society, the American Geophysical Union, and the Acoustical Society of America.

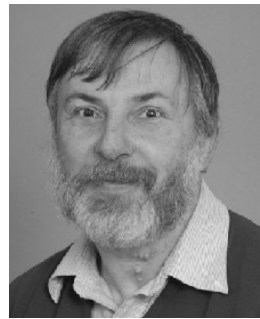

James F. Lynch (M'96-SM'03) was born in Jersey City, NJ, on June 3, 1950. He received the B.S. degree in physics from Stevens Institute of Technology, Hoboken, NJ, in 1972 and the Ph.D. degree in physics from the University of Texas, Austin, in 1978.

He was with the Applied Research Laboratories, University of Texas at Austin (ARL/UT) from 1978 to 1981 , after which he joined the scientific staff at the Woods Hole Oceanographic Institution (WHOI), Woods Hole, MA. He has been with WHOI since then and currently holds the position of Senior Scientist in the Applied Ocean Physics and Engineering Department. His research specialty areas are ocean acoustics and acoustical oceanography, but he also greatly enjoys occasional forays into physical oceanography, marine geology, and marine biology.

Dr. Lynch is a Fellow of the Acoustical Society of America and Editor-inChief of the IEEE JOURNAL OF OCEANIC ENGINEERING

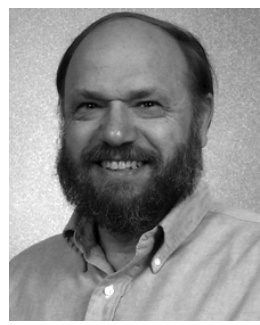

James D. Irish received the B.S. degree in physics from Antioch College, Yellow Springs, OH, in 1967 and the Ph.D. degree in oceanography from the Scripps Institution of Oceanography, La Jolla, CA, in 1971 .

He currently is a Senior Research Specialist in the Department of Applied Ocean Physics and Engineering, Woods Hole Oceanographic Institution (WHOI), Woods Hole, MA. His work has included tides, internal waves, bottom pressure observations, coastal circulation, sediment transport, acoustic instrumentation, instrument testing and evaluation, buoys and moorings, and offshore aquaculture instrumentation and engineering. His research interests include developing and deploying new instrumentation, especially with telemetry to observe the ocean.

Dr. Irish is a Member of the American Geophysical Union, the Marine Technology Society, and the Oceanographic Society.

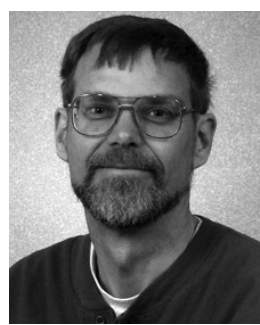

Robert C. Beardsley received the B.S degree in physics and the Ph.D. in physical oceanography from the Massachusetts Institute of Technology (MIT), Cambridge, MA, in 1964 and 1968, respectively.

He joined the Faculty, Department of Meteorology, MIT, until 1975, when he joined the Scientific Staff, Woods Hole Oceanographic Institution, Woods Hole, MA, where he now is a Senior Scientist in the Department of Physical Oceanography. He held the Walter A. and Hope Noyes Smith Chair for Excellence in Coastal Research from 1996 to 2001 and has worked on the dynamics of continental shelf circulation, with a focus on observational and numerical model studies of wind-, tidal-, and buoyancy-driven currents; mixing; and air-sea forcing on the continental shelf and marginal seas and the impact of these processes on biological processes and ecosystem dynamics.

Dr. Beardsley is a Fellow of the American Geophysical Society and of the American Association for the Advancement of Science and is a Member of the American Meteorological Society and of the Oceanography Society.

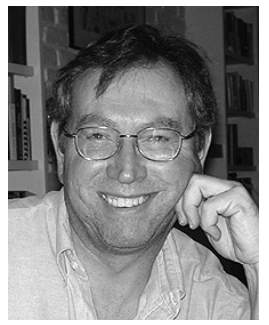

Steven R. Ramp received the M.S. degree in physical oceanography from the University of Washington, Seattle, in 1976, and the Ph.D. degree in physical oceanography from the University of Rhode Island, Narragansett, in 1986.

Since 1986, he has been with the U.S. Navy as a Professor at the Naval Postgraduate School, Monterey, CA, and a Program Officer at the Office of Naval Research, Arlington, VA. Prior to this, he spent time at the National Marine Fisheries Service, Woods Hole, MA. His research specialty is ocean observations from both ships and oceanographic moorings, and he has organized major expeditions to the Japan Sea, East China Sea, and South China Sea. He was the International Scientific Coordinator for the Asian Seas International Acoustics Experiment (ASIAEX).

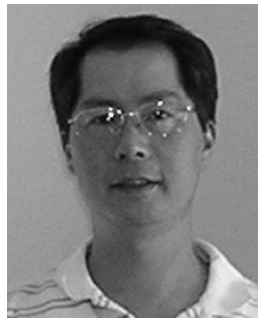

Ching-Sang Chiu received the Ph.D. degree from the Massachusetts Institute of Technology/Woods Hole Oceanographic Institution, Woods Hole, MA (MIT-WHOI) joint program in 1985.

$\mathrm{He}$ is a Professor of oceanography with the Naval Postgraduate School. His research specialties include ocean acoustics, acoustical oceanography, and coastal ocean processes and their influences on acoustics prediction. He has authored or coauthored more than 40 refereed publications in those subject areas.

Dr. Chiu is a Fellow of the Acoustical Society of America and Editor-in-Chief of the Journal of Computational Acoustics.

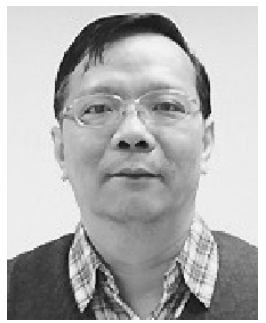

Tswen Yung Tang was born in Taiwan in 1952. He received the M.S. degree in physical oceanography from National Taiwan University, Taiwan, in 1975 and the Ph.D. degree in physical oceanography from North Carolina State University, Raleigh, in 1984.

In August 1993, he was appointed Professor of Physical Oceanography at the Institute of Oceanography, National Taiwan University. He was appointed Program Manger of Division of Marine Science, National Science Council, Taiwan, in January 2004. His research interests include equatorial dynamics, variability of upstream of Kuroshio, Kuroshio intrusion at Luzon Strait, circulation in the South China Sea, and internal waves.

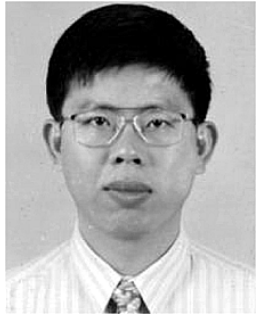

ical Union.
Ying-Jang Yang was born in Taiwan in 1967. He received the B.S. degree in oceanography and the Ph.D. degree in physical oceanography from the National Taiwan University, Taipei, Taiwan, in 1990 and 1996, respectively.

He currently is an Assistant Professor with the Department of Marine Science, Chinese Naval Academy, Kaohsiung, Taiwan. His research interests include internal tides and waves, current variation around Taiwan, and equatorial dynamics.

Dr. Yang is a Member of the American Geophys- 